\title{
On the Accurate Description of Photoionization Dynamical Parameters
}

Torsha Moitra, Aurora Ponzi, Henrik Koch, Sonia Coriani, Piero Decleva

Submitted date: 05/05/2020 - Posted date: 06/05/2020

Licence: CC BY-NC-ND 4.0

Citation information: Moitra, Torsha; Ponzi, Aurora; Koch, Henrik; Coriani, Sonia; Decleva, Piero (2020): On the Accurate Description of Photoionization Dynamical Parameters. ChemRxiv. Preprint. https://doi.org/10.26434/chemrxiv.12249755.v1

Calculation of dynamical parameters for photoionization requires an accurate description of both initial and final states of the system, as well as of the outgoing electron.

We here show, that using a linear combination of atomic orbitals (LCAO) B-spline density functional (DFT) method to describe the outgoing electron, in combination with correlated equation-of-motion coupled cluster singles and double (EOM-CCSD) Dyson orbitals, gives good agreement with experiment and outperforms other simpler approaches, like plane and Coulomb waves, used to describe the photoelectron. Results are presented for cross sections, angular distributions and dichroic parameters in chiral molecules, as well as for photoionization from excited states. We also present a comparison with the results obtained using Hartree-Fock (HF) and density-functional theory molecular orbitals selected according to Koopmans' theorem for the bound states.

File list (2) 


\title{
On the Accurate Description of
}

\section{Photoionization Dynamical Parameters}

\author{
Torsha Moitra, ${ }^{\dagger}$ Aurora Ponzi, ${ }^{\ddagger}$ Henrik Koch, ${ }^{\top}$ Sonia Coriani, ${ }^{* \dagger} \dagger$ and Piero \\ Decleva*,§ \\ $\dagger$ †TU Chemistry, Technical University of Denmark, Kemitorvet Bldg 20\%, \\ DK-2800 Kongens Lyngby, Denmark \\ $\ddagger$ Department of Physical Chemistry, Institut Ruđer Bošković, 10000 Zagreb, Croatia \\ \Scuola Normale Superiore, Piazza dei Cavalieri 7, I-56126 Pisa, Italy \\ $\S$ Dipartimento di Scienze Chimiche e Farmaceutiche, Università degli Studi di Trieste, \\ I-34121 Trieste, Italy \\ E-mail: soco@kemi.dtu.dk; decleva@units.it
}

\begin{abstract}
Calculation of dynamical parameters for photoionization requires an accurate description of both initial and final states of the system, as well as of the outgoing electron. We here show, that using a linear combination of atomic orbitals (LCAO) B-spline density functional (DFT) method to describe the outgoing electron, in combination with correlated equation-of-motion coupled cluster singles and double (EOM-CCSD) Dyson orbitals, gives good agreement with experiment and outperforms other simpler approaches, like plane and Coulomb waves, used to describe the photoelectron. Results are presented for cross sections, angular distributions and dichroic parameters in chiral molecules, as well as for photoionization from excited states. We also present a comparison with the results obtained using Hartree-Fock (HF) and density-functional theory molecular orbitals selected according to Koopmans' theorem for the bound states.
\end{abstract}


The last two decades have witnessed a remarkable increase in the study of sophisticated light-matter interactions, due to enormous advancements in synchrotron and laser sources. This is dictating high demands for reliable theoretical and computational methodologies. ${ }^{1,2}$ Photoelectron spectroscopy (PES) offers detailed information about the electronic structure of the target molecule and the properties of the ejected electrons. Photoionization has recently become important as an ultrafast probe in time-resolved experiments, both in the femto and atto second domains, as well as in the description of many strong-field phenomena. ${ }^{2-5}$ However, the interpretation of such spectroscopic signatures requires that relaxation and correlation effects in the description of bound and continuum states are taken into account. Even though, in general, the distinction between relaxation and correlation is not always well defined, we focus here on correlation effects, which are the dominant effects for valence-shell ionization. Correlation effects in the bound states and on the ionization energies have been studied extensively using several many-body approaches, like configuration interaction (CI), ${ }^{6-8}$ perturbation theory $(\mathrm{PT}),{ }^{6}$ coupled cluster (CC) ${ }^{9-13}$ and Green's functions (GF). ${ }^{6,14-16}$ Additional correlation effects are hidden in the description of the continuum, the interaction between continuum channels or between continuum and bound states.

The calculation of the photoionization observables is based on the evaluation of the dipole transition moments,

$$
\vec{D}^{i f}=\left\langle\Psi_{i}^{N}|\vec{\mu}| \Phi_{f}\right\rangle
$$

which connect the initial $\left(\Psi_{i}^{N}\right)$ and final $\left(\Phi_{f}\right)$ wave functions. The $\vec{D}^{i f}$ transition elements are linked to the differential cross section, given by (in a.u.) ${ }^{17}$

$$
\frac{d \sigma}{d \vec{k}}=4 \pi^{2} \alpha \omega\left|\vec{D}^{i f}\right|^{2}
$$

where $\alpha$ is the fine structure constant, $\omega$ is the photon energy and $\vec{k}$ is the momentum of the photoelectron in the molecular frame.

Koopmans' theorem is the simplest way to elucidate PES. The basic assumption is a 
single determinant (or single particle) description of both the initial and final states, without including orbital relaxation effects. This approximation gives a one-to-one correspondence between a molecular orbital and a spectral band, such that the ionization energy equals minus the corresponding orbital energy, $\mathrm{IE}=-\epsilon_{i}$. The model fails for ionizations involving multielectron excitations, shake-up or satellite bands. ${ }^{7,16}$

To overcome these limitations, correlation effects need to be taken into account. A key quantity in doing so is the Dyson orbital. Considering a single channel approximation of the final state $\mathrm{s}^{11,18,19}$

$$
\Phi_{f}=\Psi_{f}^{N-1} \phi_{\epsilon}
$$

the photoelectron matrix element $\vec{D}^{i f}$ can be rewritten as

$$
\vec{D}^{i f}=\left\langle\phi_{i f}^{d}|\vec{\mu}| \phi_{\epsilon}\right\rangle
$$

where $N$ is the number of electrons in the initial state, $\vec{\mu}$ is the electric dipole moment operator, $\phi_{\epsilon}$ is the wave function of the ejected electron and $\phi_{i f}^{d}$ is the Dyson orbital. The latter is defined as the overlap of the normalized wave functions for the bound $N$ and $(N-1)$ systems: ${ }^{14,20}$

$$
\phi_{i f}^{d}=\sqrt{N} \int \Psi_{i}^{N}\left(x_{1}, x_{2}, \ldots, x_{N}\right) \Psi_{f}^{N-1}\left(x_{2}, \ldots, x_{N}\right) d x_{2} \ldots d x_{N}=\sum_{p} \gamma_{p} \phi_{p}
$$

As indicated by the second equality, the Dyson orbital can be shown to reduce to a linear combination of ground-state molecular orbitals $\left(\phi_{p}\right)$, with coefficients $\gamma_{p}$. We refer to the Supporting Information for details on the derivation of Eqs. (4) and (5).

Thus, within the single channel approach, correlation effects in the bound states (both initial and final), that are in many cases the dominant ones, can be effectively incorporated via the use of the Dyson orbital approach. The nature of the Dyson orbitals acts as a marker of the effect of correlation upon changing from neutral to ionic system. It can be thought 
of as a correlated counterpart of a Hartree-Fock orbital describing the initial state of the ionized electron within Koopmans' theorem.

In this work we use a EOM-CCSD framework, ${ }^{11}$ where the left (label $L$ ) and right (label $R$ ) Dyson orbitals are distinct. ${ }^{11}$ For ionisation from the ground state, their expansion coefficients $\gamma_{p}^{L}$ (defined as $\left\langle\Psi_{L}^{N}\left|a_{p}^{\dagger}\right| \Psi_{R}^{N-1}\right\rangle$ ) and $\gamma_{p}^{R}$ (defined as $\left\langle\Psi_{L}^{N-1}\left|a_{p}\right| \Psi_{R}^{N}\right\rangle$ ) are given by:

$$
\begin{aligned}
& \gamma_{p}^{R}=\left\langle\mathrm{HF}\left|(1+\hat{\Lambda}) \exp \left(-T_{2}-T_{1}\right) a_{p}^{\dagger} R^{\mathrm{IP}} \exp \left(T_{1}+T_{2}\right)\right| \mathrm{HF}\right\rangle \\
& \gamma_{p}^{L}=\left\langle\mathrm{HF}\left|L^{\mathrm{IP}} \exp \left(-T_{2}-T_{1}\right) a_{p} \exp \left(T_{1}+T_{2}\right)\right| \mathrm{HF}\right\rangle
\end{aligned}
$$

whereas for ionisation from an excited state they are found as

$$
\begin{aligned}
& \gamma_{p}^{R}=\left\langle\mathrm{HF}\left|L^{\mathrm{EE}} \exp \left(-T_{2}-T_{1}\right) a_{p}^{\dagger} R^{\mathrm{IP}} \exp \left(T_{1}+T_{2}\right)\right| \mathrm{HF}\right\rangle \\
& \gamma_{p}^{L}=\left\langle\mathrm{HF}\left|L^{\mathrm{IP}} \exp \left(-T_{2}-T_{1}\right) a_{p} R^{\mathrm{EE}} \exp \left(T_{1}+T_{2}\right)\right| \mathrm{HF}\right\rangle
\end{aligned}
$$

In Eqs. (6)-(9), we have used the standard EOM-CCSD notation, ${ }^{12,21,22}$

$$
\begin{aligned}
\hat{\Lambda} & =\sum_{\lambda_{i}=\lambda_{1}, \lambda_{2}} \bar{t}_{\lambda_{i}} \tau_{\lambda_{i}}^{\dagger} \\
R^{\mathrm{IP}} & =\sum_{i} r_{i} a_{i}+\frac{1}{2} \sum_{a i j} r_{i j}^{a} a_{a}^{\dagger} a_{j} a_{i} \\
R^{\mathrm{EE}} & =r_{0}+\sum_{a i} r_{i}^{a} a_{a}^{\dagger} a_{i}+\frac{1}{4} \sum_{a b i j} r_{i j}^{a b} a_{a}^{\dagger} a_{b}^{\dagger} a_{j} a_{i}
\end{aligned}
$$

Having taken care of correlation effects in the bound states via, e.g., the Dyson orbital, the next step is to choose a description of the single particle continuum. We remark here that the development of computational approaches to molecular photoionization has spanned several decades. ${ }^{23-25}$ Nonetheless, full close-coupling approaches, taking in principle account of interchannel interactions, have progressed slowly, and are still computationally intensive and limited to relatively small systems. In fact, most approaches for polyatomic molecules are still at the static-exchange level, which is the counterpart for the photoelectron of the 
Koopmans description. Exceptions are the Stieltjes approach, ${ }^{23,26}$ and the equivalent Padé approach, ${ }^{27}$ that completely avoid the explicit construction of continuum states, but reconstructs cross section from purely bound pseudo-spectra. However, due to the lack of proper asymptotic boundary conditions, the separation of individual channels becomes ambiguous, and angular distributions cannot be obtained. Also, large basis sets are required and the energy resolution is quite limited. Even then, these methods have proven quite successful in combination with highly correlated electronic structure methods such as linear response coupled cluster ${ }^{28-31}$ and algebraic diagrammatic construction (ADC). ${ }^{32-34}$

The simplest approach to approximate the continuum is with plane waves $(\mathrm{PW})$ or Coulomb waves (CW), possibly orthogonalized to the Dyson orbital. ${ }^{9,35,36}$ Although this approach becomes accurate at high photoelectron energies, at least several hundred eV, it is generally rather poor at low energies, especially for angular distributions, or description of single particle continuum structures, like shape resonances, Cooper minima, and so on. A more accurate compromise, based on the muffin-tin approximation, is the multiple scattering approach. ${ }^{23}$

Another strategy is to explicitly solve the Schrödinger equation for the photoelectron wave function, which can be rather accurately done, for instance, at the static-exchange DFT level, as we do in this work. Actually, even a time-dependent TDDFT continuum can be employed, which is currently under development. An initial result will be presented here for the argon atom.

To obtain an accurate description of the continuum in large systems, a basis set approach capable of a multicentric (LCAO) description is needed. Again, several possibilities exist. ${ }^{24,37-40}$ A tricky aspect is that the description of the continuum requires basis sets which obey the particular boundary conditions of the continuum and, at the same time, reproduce the oscillating behaviour of the photoelectron wave function to infinity. Conventional Gaussian- or Slater-type orbitals are unsuitable for this purpose. B-spline functions, on the other hand, are quite useful in this regards. They are very flexible, they accurately 
describe both the bound and continuum states with minimal numerical dependencies, ${ }^{41}$ and they are becoming popular for continuum calculations. ${ }^{38,40,42}$

Here, we employ a LCAO B-spline approach where the primitive basis functions $\chi_{j l m}$ are expressed as product of radial, $B_{j}(r)$, and angular $Y_{l m}(\theta, \phi)$ functions,

$$
\chi_{j l m}=\frac{1}{r} B_{j}(r) Y_{l m}(\theta, \phi)
$$

A long-range, large angular momentum basis is centred on the origin, to describe the asymptotic part. Much smaller expansions are employed instead at the nuclear positions, to take care of the Coulomb cusps, and to avoid linear dependence. Converged results are easily obtained even for quite large systems and heavy atoms.

The continuum orbitals are computed by solving the Schrödinger equation in the angular momentum representation

$$
h_{K S} \phi_{\epsilon, l m}=E \phi_{\epsilon, l m}
$$

with real (K-matrix) boundary conditions. In the Hamiltonian operator

$$
h_{K S}=-\frac{1}{2} \Delta+V_{N}+V_{C}+V_{X C}
$$

the term $V_{N}, V_{C}$ and $V_{X C}$ are the nuclear attraction, Coulomb and exchange-correlation potentials, respectively.

Angular momentum analysis of the differential photoionization cross-section in Eq. (2) gives

$$
\frac{d \sigma}{d k}=\frac{\sigma}{4 \pi}\left[1+\left(-\frac{1}{2}\right)^{\left|m_{r}\right|} \beta P_{2}(\cos \theta)+m_{r} D P_{1}(\cos \theta)\right]
$$

This shows that the angular distribution of the photoelectron in the solid angle $d k$ along the direction of propagation $(k)$ can be completely defined by three parameters: the crosssection $\sigma$, the asymmetry parameter $\beta$ and the dichroism parameter $D$. Here, $P_{i}$ is a Legendre 
polynomial of order $i$, whereas $\theta$ is the scattering angle between $k$ and the laboratory frame defined by the polarization or the direction of propagation of the light. The parameter $m_{r}$ specifies the light polarization, with three possible values, $0,+1$ and -1 , for linearly polarized (LP), left circularly polarized (LCP) and right circularly polarized (RCP) light, respectively. For LP, Eq. (16) assumes a very simple form,

$$
\frac{d \sigma}{d k}=\frac{\sigma}{4 \pi}\left[1+\beta P_{2}(\cos \theta)\right]
$$

The details of the incorporation of EOM-CCSD Dyson orbital coefficients to obtain the differential cross-section in Eq. (16) can be found in the Supporting Information.

In this letter, we present a combined EOM-CCSD Dyson-orbital/DFT continuum approach for the photoionization observables, and compare our results with both experiments and other computational approaches, including $\mathrm{PW}$ or $\mathrm{CW}$ results. In more detail, we compare the partial cross-sections and asymmetry parameters for atomic (Ar) and small molecular $\left(\mathrm{CO}, \mathrm{H}_{2} \mathrm{O}\right.$, CS and $\left.\mathrm{CH}_{2} \mathrm{O}\right)$ systems, for which reliable experimental or literature data are available, by treating the bound and continuum parts at different levels of theorynamely: HF molecular orbitals, DFT molecular orbitals and EOM-CCSD Dyson orbitals for the bound state and single-center and multi-center approaches for the continuum state. The dichroic parameter, as an example of photoionization of chiral molecules, is presented for methyloxirane. We also compute the photoionization dynamical parameters from the lowest lying excited states of furan, as accessible in pump-probe experiments, ${ }^{2,3}$ which can be taken up by experimentalists for further spectroscopic studies. Our study takes into account the individual pathways contributing towards the photoionization dynamical parameters. This gives a clear picture of the dominantly contributing ionizations of the system.

For most of the molecules, experimental geometries from the NIST database were used. ${ }^{43}$ The geometry of $(S)$-methyloxirane was taken from previous work, ${ }^{44}$ and so was the geometry used for furan. ${ }^{45}$ 
The EOM-CCSD Dyson orbitals were computed using Q-Chem ${ }^{11,46}$ and the aug-cc-pVTZ basis set. PW and CW cross-sections and asymmetry parameters were computed using the ezDyson code. ${ }^{47}$ As anticipated, we adapted the LCAO B-spline code ${ }^{42}$ in order to take into account the different left and right Dyson orbital coefficients. (See the discussion in the Supporting Information). To compute the continuum functions, ground state electronic densities were obtained from ADF using the LB94 exchange-correlation potential and the DZP basis from the ADF database. ${ }^{48}$ The choice of the functional was guided by literature studies showing its good performance for the calculation of photoionization observables. ${ }^{49,50}$ All parameters used to calculate the observables are listed in Table S1 and S2. Notice that we present the results according to conventional symmetry labelling, even though the QChem calculations adopted non-Mulliken symmetry convention. ${ }^{46}$

The performance of the various combinations of methods here employed to describe bound and continuum states was assessed for the three lowest-energy ionizations of $\mathrm{CO}, \mathrm{H}_{2} \mathrm{O}$ and $\mathrm{CH}_{2} \mathrm{O}$ and for the two lowest of CS. Fig. 1 shows the results for $\mathrm{CO}$ and $\mathrm{H}_{2} \mathrm{O}$. The results for $\mathrm{CS}$ and $\mathrm{CH}_{2} \mathrm{O}$ are documented in the Supporting Information (see Fig. S3-S6), due to lack of experimental data and to avoid repetition of results.

In general, the B-spline DFT method for the continuum in conjunction with the EOMCCSD Dyson orbitals offers the best description of photoionization processes, amongst the various combinations here considered. For $\mathrm{CO}$ and $\mathrm{H}_{2} \mathrm{O}$, there is very little difference between the results obtained from the EOM-CCSD correlated Dyson orbitals and those from the Koopmans theorem-based HF and DFT MOs. This is quite reasonable, given that the major contributing MO in the Dyson orbital has a coefficient greater than 0.9 for the corresponding HF or DFT MO. A slight vertical deviation is seen for our method with respect to experiment for the third lowest-lying ionization (the ionization to $\mathrm{B}{ }^{2} \Sigma^{+}$) of CO. Even though the PW and CW approximations often reproduce the spectral profile, it shows non-uniform large vertical shifts. The discrepancy is larger near the ionization threshold. This is justifiable as PW and CW are two extreme approximations and the actual charge on the ionized core is 

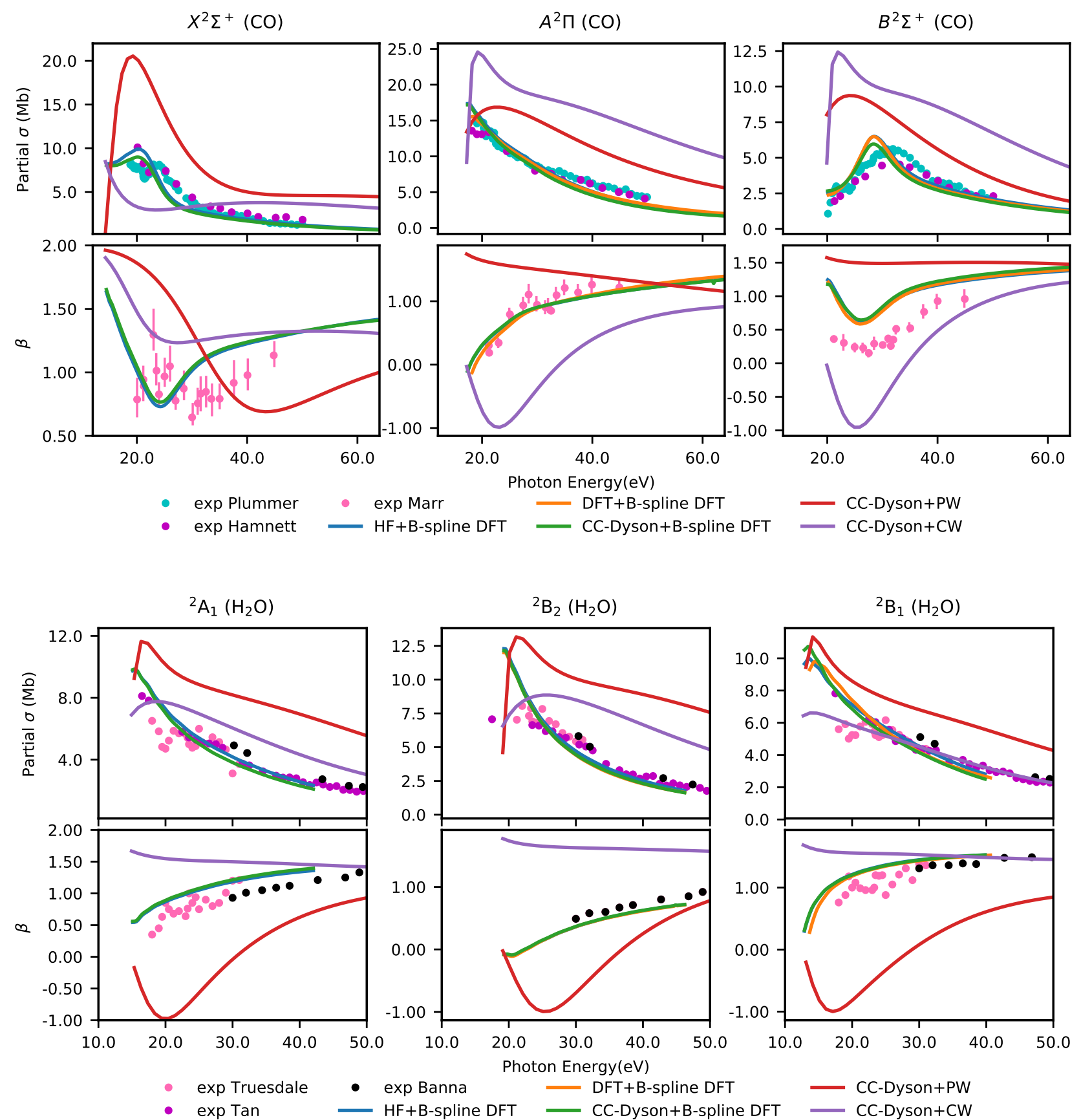

Figure 1: Partial cross-section and asymmetry parameters of $\mathrm{CO}$ and $\mathrm{H}_{2} \mathrm{O}$ relative to the three lowest energy ionizations. Experimental result taken from Ref. 51, 52, 53, 54, 55 and 56 .

some value in the range $[0,1] .{ }^{9}$

The Cooper minimum is a spectral feature observed in the photoionization cross-section 
of $\mathrm{Ar}$ at around $47 \mathrm{eV}$. It is a signature of the sign change in the photoelectron matrix element as the outgoing d-wave moves towards higher kinetic energy. Both the shape of the Dyson orbital and the description of the outgoing electron define the shape of the Cooper minimum, see Fig. 2. As previously ascertained, ${ }^{9}$ the $\mathrm{PW}$ and $\mathrm{CW}$ descriptions of the

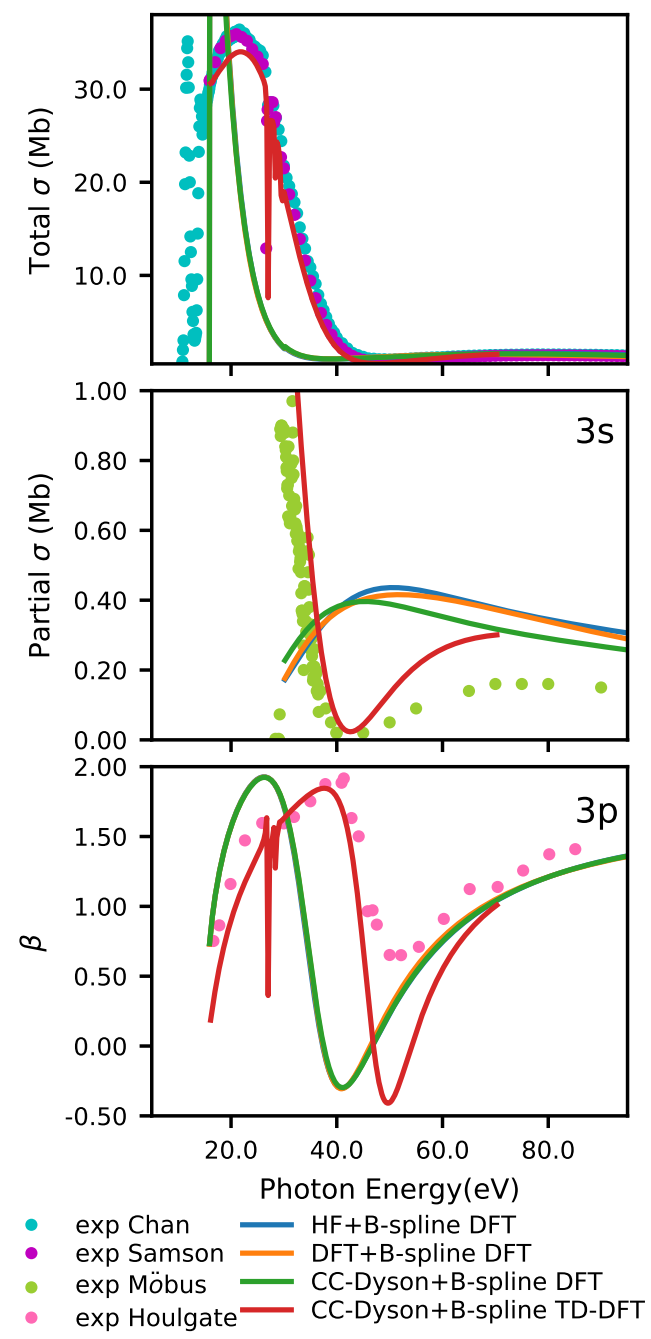

Figure 2: Total and partial cross section and asymmetry parameter of Ar calculated with HF, DFT and EOM-CCSD methods. Multicenter B-spline DFT and TD-DFT functions are used to describe the continuum. The calculated results with DFT B-spline continuum are overlapping. Experimental results are from Refs. 57, 58, 59 and 60.

photoelectron wave function fail to reproduce the Cooper minimum (see also Fig. S1). The spectral profile of the total cross-section is reproduced using the B-spline DFT technique for the continuum, even though the minimum is shifted by about $15 \mathrm{eV}$. It is seen to be a feature 
arising due to ionization from the $3 \mathrm{p}$ orbital of Ar. As the magnitude of the partial crosssection of ionization from $3 \mathrm{p}$ orbital is much larger than that of $3 \mathrm{~s}$, the total cross-section is very similar to the partial cross-section from 3p orbital (as shown in Fig. S1). Electronelectron interaction mixes the $3 \mathrm{~s}$ and $3 \mathrm{p}$ channels. As the $3 \mathrm{p}$ cross-section is much larger, the Cooper minimum is also felt in the 3s ionization. However, the DFT B-spline continuum approach lacks correlation in the treatment of the continuum and thus does not yield the minimum in the partial cross-section of ionization from the 3 s orbital. The time-dependent (TD)DFT description of the continuum overcomes this problem and provides a remarkable agreement with experiment, as it can be appreciated from Fig. 2. Notable improvement in the asymmetry parameter corresponding to ionization from the $3 p$ orbital is also seen upon consideration of TD-DFT B-spline continuum. The minimum is obtained at the correct photon energy, whereas the dip in the spectral feature is overestimated by about $1 \beta$ unit.

The photoelectron dichroism parameter $(D)$ is governed by the description of the nature of the ionized state. From now onwards, we do not report on the PW and CW descriptions of the continuum, having assessed their inadequacy to properly calculate photoionization dynamical variables.

Fig. 3 illustrates our approach on the $D$ of $(S)$-methyloxirane. As immediately apparent, correlation effects must be accounted for while describing the bound state of the target. Koopmans' theorem fails completely to reproduce the experimental observations, especially in the near-threshold region which usually acts as a fingerprint region. The HF-based $D$ results (blue curves in Fig. 3) show large deviations from experiment or are completely opposite in sign. DFT molecular orbitals perform better. EOM-CCSD Dyson orbitals coupled with B-spline DFT give spectral features within the experimental error bar. Also, it is observed that the near threshold results are most sensitive to the bound state description.

Finally, we studied the ground and excited state dynamics of photoionization of furan, see Fig. 4. At the ground-state equilibrium geometry, the HOMO and HOMO-1 orbitals are $1 \mathrm{a}_{2}$ and $2 \mathrm{~b}_{1} \pi$-orbitals, respectively, which leads to two well resolved bands in PES. For 


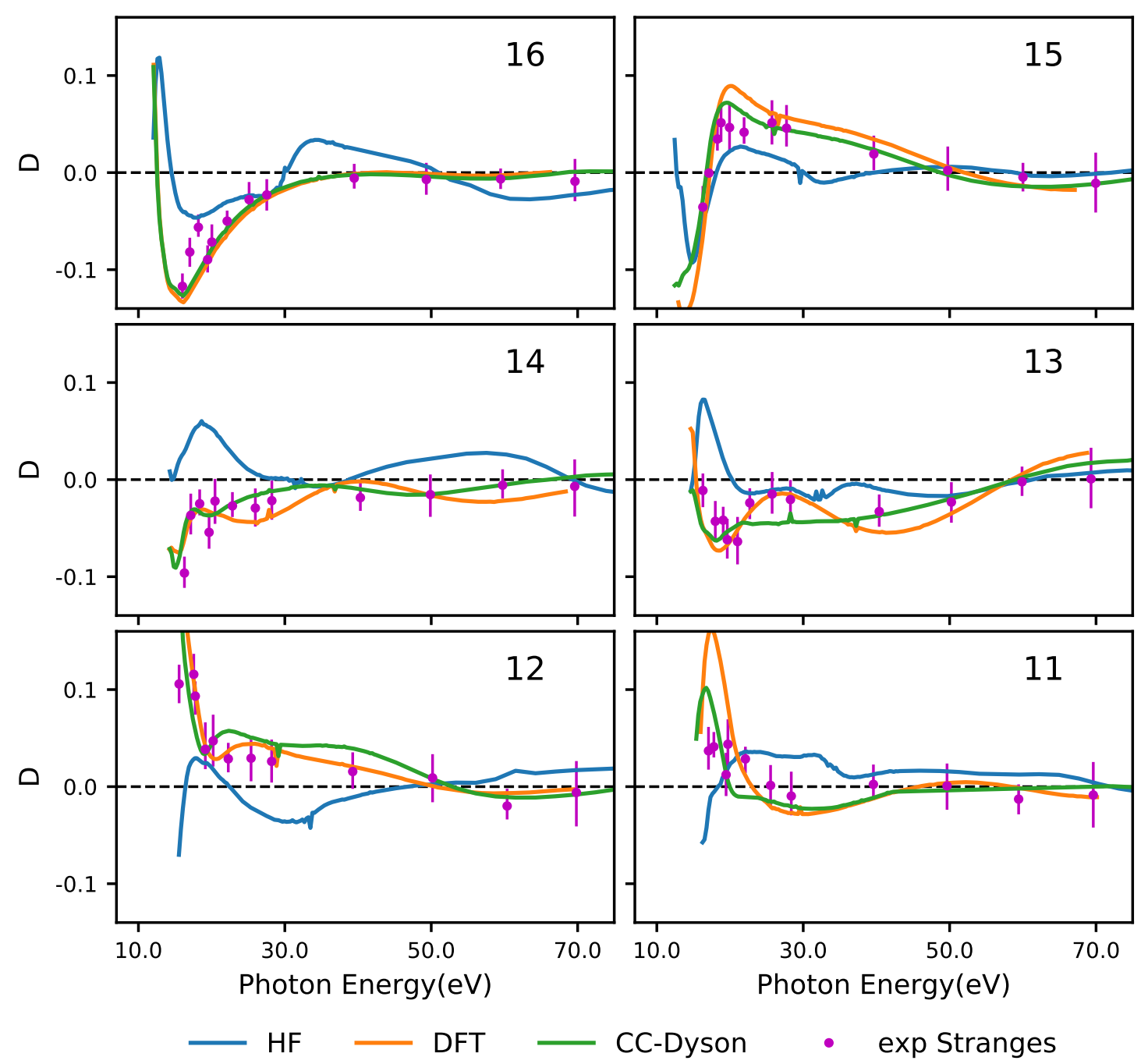

Figure 3: Photoelectron circular dichroism parameter $D$ of $(S)$-methyloxirane $\left(\mathrm{C}_{3} \mathrm{H}_{6} \mathrm{O}\right)$ calculated with B-spline DFT functions to represent the outgoing electron and three different electronic structure methods for the bound states. Experimental results are taken from Ref. 61. The numerical label in each subfigure indicates the MO from which the ionization predominantly occurs.

the ground state ionizations, the partial cross-sections are steeply decreasing with no other significant feature. On the other hand, the experimental anisotropy parameters $\beta$ increase rapidly near the threshold and become constant at about $50 \mathrm{eV}$. The same trend is roughly observed in the computed $\beta$. The two lowest-lying excited states of furan are the ${ }^{1} \mathrm{~A}_{2}$ and ${ }^{1} \mathrm{~B}_{2}$ states. Transition to ${ }^{1} \mathrm{~A}_{2}$ state is dark and is of Rydberg $(\pi 3 \mathrm{~s})$ character, whereas ${ }^{1} \mathrm{~B}_{2}$ is a bright state. We considered the ionization from these two excited states to the lowest-energy 


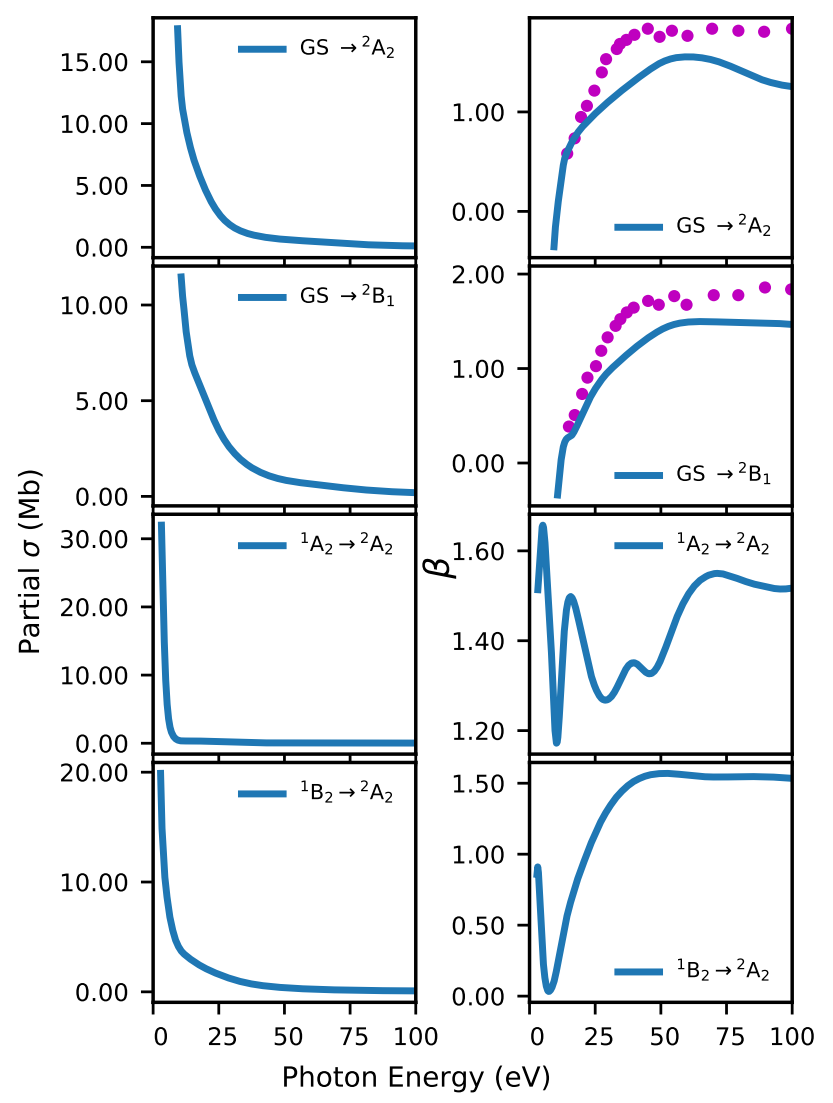

Figure 4: Partial cross-section and asymmetry parameter from ground and lowest lying excited states of furan $\left(\mathrm{C}_{4} \mathrm{H}_{4} \mathrm{O}\right)$ using ground state equilibrium geometry. EOM-CCSDDyson with multicenter B-Spline DFT continuum. Experimental results (shown by dots) are taken from Ref. 62.

ionized state ( ${ }^{2} \mathrm{~A}_{2}$ in both cases). The dark state is characterized by excitation from occupied $1 \mathrm{a}_{2}$ to virtual $10 \mathrm{a}_{1}$. However, the bright state is a combination of transition from $1 \mathrm{a}_{2}$ to $3 \mathrm{~b}_{1}$ and 5b $\mathrm{b}_{1}$ MO's. The square of the Dyson norm (here computed as $\left|\phi^{L} \cdot \phi^{R}\right|$ ) corresponding to ionization from both excited states is about 0.48 . This value, close to 0.5 , reflects ionization from a singly occupied orbital in the initial state.

For both ground and excited state ionization processes, $\beta$ is more sensitive than partial $\sigma$ and shows an initial decrease followed by gradual increase, finally being constant at around $1.5 \beta$ units. A steep descent in $\sigma$ is observed in all cases, where the steepness is a measure of the diffuseness of the orbital from which ionization occurs.

To summarize, our methodology - using EOM-CCSD Dyson orbital coefficients to de- 
scribe the bound initial and final states of the target system and B-spline DFT functions to describe the continuum - gives an accurate description of photoionization dynamic properties. On the other hand, simple PW or CW continuum results appear too erratic to be of much use at low electron kinetic energy.

The present approach is completely general, allowing the calculation of all photoionization observables, including, although not considered in this initial study, the photoionization from molecules aligned or fixed in space. This contrasts Stieltjes or Padé pure bound state approaches, which, although useful in limited contexts, lack sufficient generality. With the DFT B-spline continuum, only interchannel coupling effects, which are less widespread, lie outside our formulation. However, as our preliminary results illustrate in the case of argon, the use of the TD-DFT continuum may alleviate this deficiency. Further theoretical improvements include application to core ionizations by coupling the bound state calculation with the core-valence-separation (CVS) approach, ${ }^{63-65}$ and the description of multielectron excitations, i.e. shake-up states, which will require the inclusion of higher-order (beyond CCSD) correlation effects for an accurate description of the bound states.

Finally, the approach is computationally quite efficient and could be used for the description of nuclear motion problems, like vibrationally resolved spectra or imaging of nuclear trajectories, which require a large number of individual calculations. Besides being an always open channel if the photon energy is high enough, its ultrafast response and its high sensitivity to the nature of the orbital from which the electron is ionized make photoionization an ideal probe in time resolved experiments, as well as a source of coherent electronic wavepacket preparation in attosecond experiments.

\section{Acknowledgement}

The authors thank Marta Lopez Vidal for help in setting up some of the Q-Chem calculations. T. M., H. K. and S. C. acknowledge support from the Marie Skłodowska-Curie European 
Training Network "COSINE-COmputational Spectroscopy In Natural sciences and Engineering", Grant Agreement No. 765739. H.K. and S.C. acknowledge the Research Council of Norway through FRINATEK project 275506, TheoLight. S. C. acknowledges support from the Independent Research Fund Denmark, DFF-RP2 grant no. 7014-00258B. This research has also been supported by the Croatian Science Foundation under the grant HRZZ IP-2016-06-1142. Finally, the COST Action CA18222, Attochem, is acknowledged.

\section{Supporting Information Available}

The Supporting Information is available free of charge.

(1) Definitions; (2) Derivation of Eq. (4); (3) Photoelectron transition strength in the EOMCC formalism; (4) Calculation of photoionization observables using the LCAO B-spline approach, (5) Parameters used in the LCAO-B-spline code and in the ezDyson code, (6) Ionization energies and Dyson norms; (7) Partial cross-sections and asymmetry parameters; (8) Excitation and Ionization energies of Furan; (9) Excited state cross-section and asymmetry parameter of furan at bent geometry; (10) Cartesian coordinates for all molecules considered.

\section{References}

(1) Mobilio, S., Boscherini, F., Meneghini, C., Eds. Synchrotron Radiation: Basics, Methods and Applications; Springer, 2014.

(2) Suzuki, T. Time-resolved photoelectron spectroscopy of non-adiabatic electronic dynamics in gas and liquid phases. Int. Reviews Phys. Chem. 2012, 31, 265-318.

(3) Stolow, A.; Underwood, J. G. Time-Resolved Photoelectron Spectroscopy of Nonadiabatic Dynamics in Polyatomic Molecules. In Adv. Chem. Phys.; John Wiley \& Sons, Ltd, 2008; Chapter 6, pp 497-584. 
(4) Nisoli, M.; Decleva, P.; Calegari, F.; Palacios, A.; Martín, F. Attosecond Electron Dynamics in Molecules. Chem. Rev. 2017, 117, 10760-10825.

(5) Wolf, T. J. A.; Parrish, R. M.; Myhre, R. H.; Martínez, T. J.; Koch, H.; Gühr, M. Observation of Ultrafast Intersystem Crossing in Thymine by Extreme Ultraviolet TimeResolved Photoelectron Spectroscopy. J. Phys. Chem. A 2019, 123, 6897-6903.

(6) Szabo, A.; Ostlund, N. S. Modern Quantum Chemistry: Introduction to Advanced Electronic Structure Theory; Mineola, N.Y.: Dover Publications, 1996.

(7) Bawagan, A. D.; Davidson, E. R. Understanding electron correlation: Recent progress in molecular synchrotron photoelectron spectroscopy. Adv. Chem. Phys. 1999, 110, 215-263.

(8) Arneberg, R.; Müller, J.; Manne, R. Configuration interaction calculations of satellite structure in photoelectron spectra of $\mathrm{H}_{2} \mathrm{O}$. Chem. Phys. 1982, 64, $249-258$.

(9) Gozem, S.; Gunina, A. O.; Ichino, T.; Osborn, D. L.; Stanton, J. F.; Krylov, A. I. Photoelectron Wave Function in Photoionization: Plane Wave or Coulomb Wave? J. Phys. Chem. Lett. 2015, 6, 4532-4540.

(10) Oana, C. M.; Krylov, A. I. Cross sections and photoelectron angular distributions in photodetachment from negative ions using equation-of-motion coupled-cluster Dyson orbitals. J. Chem. Phys. 2009, 131, 124114.

(11) Oana, C. M.; Krylov, A. I. Dyson orbitals for ionization from the ground and electronically excited states within equation-of-motion coupled-cluster formalism: Theory, implementation, and examples. J. Chem. Phys. 2007, 127, 234106.

(12) Bartlett, R. J. Coupled-cluster theory and its equation-of-motion extensions. WIREs Comput. Mol. Sci. 2012, 2, 126-138. 
(13) Bartlett, R. J.; Musiał, M. Coupled-cluster theory in quantum chemistry. Rev. Mod. Phys. 2007, 79, 291-352.

(14) Ortiz, J. Toward an Exact One-Electron Picture of Chemical Bonding. In Advances in Quantum Chemistry; Löwdin, P.-O., Ed.; Academic Press, 1999; Vol. 35; pp 33-52.

(15) Cederbaum, L. S.; Domcke, W. Theoretical aspects of ionization potentials and photoelectron spectroscopy: A Green's function approach. Adv. Chem. Phys 1977, 36, 205.

(16) Cederbaum, L. S.; Domcke, W.; Schirmer, J.; Vonniessen, W. Correlation effects in the ionization of molecules: Breakdown of the molecular orbital picture. Adv. Chem. Phys 1986, 65, 115-159.

(17) Bethe, H. A.; Salpeter, E. E. Quantum Mechanics of One- and Two-Electron Atoms; Plenum: New York, 1977.

(18) Chandra, N. Photoelectron spectroscopic studies of polyatomic molecules. I. Theory. J. Phys. B: At. Mol. Phys. 1987, 20, 3405-3415.

(19) Ponzi, A.; Angeli, C.; Cimiraglia, R.; Coriani, S.; Decleva, P. Dynamical photoionization observables of the CS molecule: The role of electron correlation. J. Chem. Phys. 2014, $140,204304$.

(20) Martin, R. L.; Shirley, D. A. Theory of core-level photoemission correlation state spectra. J. Chem. Phys. 1976, 64, 3685-3689.

(21) Stanton, J. F.; Bartlett, R. J. The equation of motion coupled-cluster method. A systematic biorthogonal approach to molecular excitation energies, transition probabilities, and excited state properties. J. Chem. Phys. 1993, 98, 7029-7039. 
(22) Krylov, A. I. Equation-of-Motion Coupled-Cluster Methods for Open-Shell and Electronically Excited Species: The Hitchhiker's Guide to Fock Space. Ann. Rev. Phys. Chem. 2008, 59, 433-462.

(23) Rescigno, T.; McKoy, V.; Schneider, B. Electron-Molecule and Photon-Molecule Collisions; Plenum Press: New York, 1979.

(24) Gianturco, F.; Huo, W. Computational Methods for Electron-Molecule Collisions; Springer: US, 1995.

(25) Burke, P. G. R-matrix theory of atomic collisions: application to atomic, molecular and optical processes; Springer, 2011.

(26) Müller-Plathe, F.; Diercksen, G. H. Molecular photoionisation cross sections by moment theory. An introduction. In Electronic Structure of Atoms, Molecules and Solids. Proceeding of the II Escola Brasileira de Estructure Eletronica, Olinda, Brazil, July 1722, 1989; Canuto, S., D’Albuquerque e Castro, J., Paixao, F. J., Eds.; World Scientific: Olinda, Brazil, 1990; pp 1-29.

(27) Nascimento, M. A. C. Calculation of photionization cross-sections and dynamic polarizabilities using square integrable basis sets and correlated wave functions. J. Mol. Struc.: THEOCHEM 1985, 120, $227-240$.

(28) Cukras, J.; Coriani, S.; Decleva, P.; Christiansen, O.; Norman, P. Photoionization cross section by Stieltjes imaging applied to coupled cluster Lanczos pseudo-spectra. J. Chem. Phys. 2013, 139, 094103.

(29) Cukras, J.; Decleva, P.; Coriani, S. A coupled-cluster study of photodetachment cross sections of closed-shell anions. J. Chem. Phys. 2014, 141, 174315.

(30) Tenorio, B. N. C.; Nascimento, M. A. C.; Coriani, S.; Rocha, A. B. Coupled Clus- 
ter Study of Photoionization and Photodetachment Cross Sections. J. Chem. Theory Comput. 2016, 12, 4440-4459.

(31) Tenorio, B. N. C.; Moitra, T.; Nascimento, M. A. C.; Rocha, A. B.; Coriani, S. Molecular inner-shell photoabsorption/photoionization cross sections at core-valence-separated coupled cluster level: Theory and examples. J. Chem. Phys. 2019, 150, 224104.

(32) Neville, S. P.; Averbukh, V.; Ruberti, M.; Yun, R.; Patchkovskii, S.; Chergui, M.; Stolow, A.; Schuurman, M. S. Excited state X-ray absorption spectroscopy: Probing both electronic and structural dynamics. J. Chem. Phys. 2016, 145, 144307.

(33) Ruberti, M.; Yun, R.; Gokhberg, K.; Kopelke, S.; Cederbaum, L. S.; Tarantelli, F.; Averbukh, V. Total Molecular Photoionization Cross- Sections by Algebraic Diagrammatic Construction-Stieltjes-Lanczos method: Benchmark Calculations. J. Chem. Phys. 2013, 139, 144107.

(34) Averbukh, V.; Cederbaum, L. S. Ab initio calculation of interatomic decay rates by a combination of the Fano ansatz, Green's-function methods, and the Stieltjes imaging technique. J. Chem. Phys. 2005, 123, 204107.

(35) Humeniuk, A.; Wohlgemuth, M.; Suzuki, T.; Mitrić, R. Time-resolved photoelectron imaging spectra from non-adiabatic molecular dynamics simulations. J. chem. Phys. 2013, 139, 134104.

(36) Brumboiu, I. E.; Eriksson, O.; Norman, P. Photoelectron Spectroscopy of Molecules Beyond the Electric Dipole Approximation. J. Chem. Theory Comput. 2019, 15, 54835494.

(37) Lucchese, R. R.; Takatsuka, K.; McKoy, V. Applications of the Schwinger variational principle to electron-molecule collisions and molecular photoionization. Phys. Rep. 1986, $131,147-221$. 
(38) Mašín, Z.; Benda, J.; Gorfinkiel, J. D.; Harvey, A. G.; Tennyson, J. UKRmol+: A suite for modelling electronic processes in molecules interacting with electrons, positrons and photons using the R-matrix method. Comput. Phys. Commun. 2020, 249, 107092.

(39) Majety, V. P.; Zielinski, A.; Scrinzi, A. Photoionization of few electron systems: a hybrid coupled channels approach. New J. Phys. 2015, 17, 063002.

(40) Marante, C.; Klinker, M.; Corral, I.; González-Vázquez, J.; Argenti, L.; Martín, F. Hybrid-Basis Close-Coupling Interface to Quantum Chemistry Packages for the Treatment of Ionization Problems. J. Chem. Theory Comput. 2017, 13, 499-514, PMID: 28058835 .

(41) Bachau, H.; Cormier, E.; Decleva, P.; Hansen, J. E.; Martín, F. Applications of Bsplines in atomic and molecular physics. Rep. Prog. Phys 2001, 64, 1815-1943.

(42) Toffoli, D.; Stener, M.; Fronzoni, G.; Decleva, P. Convergence of the multicenter Bspline DFT approach for the continuum. Chem. Phys. 2002, 276, 25 - 43.

(43) Linstrom, P.; Mallard, W. NIST Chemistry WebBook, NIST Standard Reference Database. 2017; http://webbook.nist.gov.

(44) Di Tommaso, D.; Stener, M.; Fronzoni, G.; Decleva, P. Conformational Effects on Circular Dichroism in the Photoelectron Angular Distribution. ChemPhysChem 2006, 7, 924-934.

(45) Ponzi, A.; Sapunar, M.; Angeli, C.; Cimiraglia, R.; Došlić, N.; Decleva, P. Photoionization of furan from the ground and excited electronic states. J. Chem. Phys. 2016, 144, 084307.

(46) Shao, Y. et al. Advances in molecular quantum chemistry contained in the Q-Chem 4 program package. Mol. Phys. 2015, 113, 184-215.

(47) Gozem, S.; Krylov, A. ezDyson. version 4.0 (accessed February 2020). 
(48) Van Lenthe, E.; Baerends, E. J. Optimized Slater-type basis sets for the elements 1âĂŞ118. J. Comput. Chem. 2003, 24, 1142-1156.

(49) van Leeuwen, R.; Baerends, E. J. Exchange-correlation potential with correct asymptotic behavior. Phys. Rev. A 1994, 49, 2421-2431.

(50) Stener, M.; Furlan, S.; Decleva, P. Density functional calculations of photoionization with an exchange-correlation potential with the correct asymptotic behaviour. J. Phys. B-At. Mol. Opt. 2000, 33, 1081-1102.

(51) Plummer, E. W.; Gustafsson, T.; Gudat, W.; Eastman, D. E. Partial photoionization cross sections of $\mathrm{N}_{2}$ and $\mathrm{CO}$ using synchrotron radiation. Phys. Rev. A 1977, 15, 23392355.

(52) Hamnett, A.; Stoll, W.; Brion, C. Photoelectron branching ratios and partial ionization cross-sections for $\mathrm{CO}$ and $\mathrm{N}_{2}$ in the energy range 18-50 eV. J Electron Spectros. Relat. Phenomena 1976, 8, 367-376.

(53) Marr, G. V.; Morton, J. M.; Holmes, R. M.; McCoy, D. G. Angular distribution of photoelectrons from free molecules of $\mathrm{N}_{2}$ and $\mathrm{CO}$ as a function of photon energy. $J$. Phys. B - At. Mol. Opt. 1979, 12, 43-52.

(54) Truesdale, C. M.; Southworth, S.; Kobrin, P. H.; Lindle, D. W.; Thornton, G.; Shirley, D. A. Photoelectron angular distributions of $\mathrm{H}_{2}$ O. J. Chem. Phys. 1982, 76, 860-865.

(55) Banna, M. S.; McQuaide, B. H.; Malutzki, R.; Schmidt, V. The photoelectron spectrum of water in the 30 to $140 \mathrm{eV}$ photon energy range. J. Chem. Phys. 1986, 84, 4739-4744.

(56) Tan, K.; Brion, C.; der Leeuw, P. V.; van der Wiel, M. Absolute oscillator strengths (10-60 eV) for the photoabsorption, photoionisation and fragmentation of $\mathrm{H}_{2} \mathrm{O}$. Chem. Phys. 1978, 29, $299-309$. 
(57) Samson, J.; Stolte, W. Precision measurements of the total photoionization crosssections of He, Ne, Ar, Kr, and Xe. J Electron Spectros. Relat. Phenomena 2002, 123, 265-276.

(58) Chan, W. F.; Cooper, G.; Guo, X.; Burton, G. R.; Brion, C. E. Absolute optical oscillator strengths for the electronic excitation of atoms at high resolution. III. The photoabsorption of argon, krypton, and xenon. Phys. Rev. A 1992, 46, 149-171.

(59) Möbus, B.; Magel, B.; Schartner, K.-H.; Langer, B.; Becker, U.; Wildberger, M.; Schmoranzer, H. Measurements of absolute Ar 3s photoionization cross sections. Phys. Rev. A 1993, 47, 3888-3893.

(60) Houlgate, R.; West, J.; Codling, K.; Marr, G. The angular distribution of the 3p electrons and the partial cross section of the $3 \mathrm{~s}$ electrons of argon from threshold to $70 \mathrm{eV}$. J Electron Spectros Relat Phenomena 1976, 9, 205 - 209.

(61) Stranges, S.; Turchini, S.; Alagia, M.; Alberti, G.; Contini, G.; Decleva, P.; Fronzoni, G.; Stener, M.; Zema, N.; Prosperi, T. Valence photoionization dynamics in circular dichroism of chiral free molecules: The methyl-oxirane. J. Chem. Phys. 2005, 122, 244303.

(62) Keller, P.; Taylor, J.; Carlson, T.; Grimm, F. A systematic angle-resolved photoelectron study of $\pi$ orbitals in unsaturated organic molecules. J. Electron Spectros. Relat. Phenomena 1984, 33, $333-345$.

(63) Cederbaum, L. S.; Domcke, W.; Schirmer, J. Many-body theory of core holes. Phys. Rev. A: At. Mol. Opt. Phys. 1980, 22, 206-222.

(64) Coriani, S.; Koch, H. Communication: X-ray Absorption Spectra and Core-Ionization Potentials within a Core-Valence Separated Coupled Cluster Framework. J. Chem. Phys. 2015, 143, 181103, Ibidem 2016, 145, 149901. 
(65) Vidal, M. L.; Feng, X.; Epifanovsky, E.; Krylov, A. I.; Coriani, S. New and Efficient Equation-of-Motion Coupled-Cluster Framework for Core-Excited and Core-Ionized States. J. Chem. Theory Comput. 2019, 15, 3117-3133. 


\section{Graphical TOC Entry}

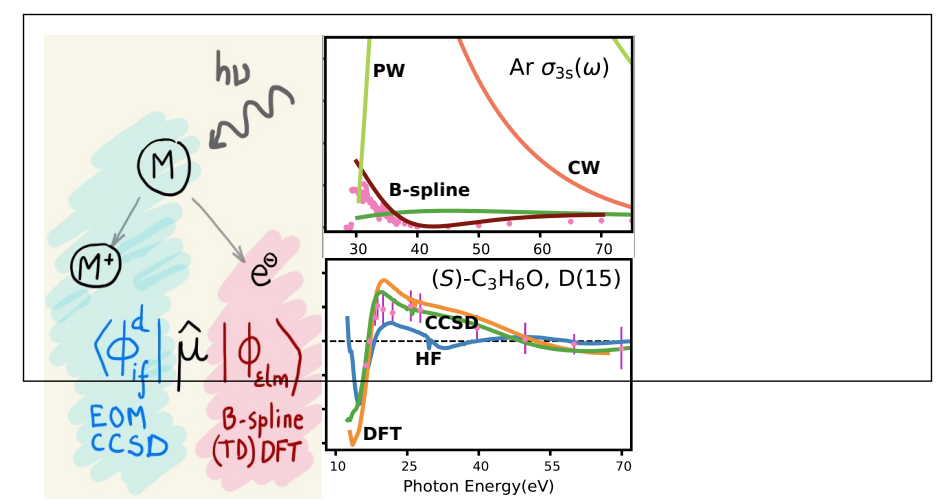




\section{On the Accurate Description of}

\section{Photoionization Dynamical Parameters. Supporting Information}

Torsha Moitra, $^{\dagger}$ Aurora Ponzi, ${ }^{\ddagger}$ Henrik Koch, " Sonia Coriani, ${ }^{*} \dagger$ and Piero Decleva* $^{* \S}$

$\dagger$ †TU Chemistry, Technical University of Denmark, DK-2800 Kongens Lyngby, Denmark

$\ddagger$ Department of Physical Chemistry, Institut Ruđer Bošković, 10000 Zagreb, Croatia

9Scuola Normale Superiore, Piazza dei Cavalieri 7, I-56126 Pisa, Italy

$\S$ Dipartimento di Scienze Chimiche e Farmaceutiche, Università degli Studi di Trieste, I-34121 Trieste, Italy

E-mail: soco@kemi.dtu.dk; decleva@units.it 


\section{Definitions}

Our objective is to compute the photoelectron transition moments between an initial (label $I)$ and a final (label $F$ ) state

$$
D^{F I}=\left\langle\Psi_{F}|\hat{\mu}| \Psi_{I}\right\rangle
$$

where $\hat{\mu}$ is the dipole moment operator.

Following the works of Arneberg et al., ${ }^{1}$ we write the final state $\left(\Psi_{F}\right)$ for the photoionization process as an antisymmetrized product of the wavefunction of the photoelectron (the continuum electron), $\phi_{\epsilon}$, and a bound $(N-1)$-electron wavefunction $\Psi^{N-1}$ :

$$
\Psi_{F} \equiv \Psi_{F \epsilon}=\mathcal{A}\left(\Psi_{F}^{N-1} \phi_{\epsilon}\right)=a_{\epsilon}^{\dagger} \Psi_{F}^{N-1}
$$

The (transition) dipole operator in second quantization is

$$
\hat{\mu}=\sum_{p q} \mu_{p q} a_{p}^{\dagger} a_{q}
$$

where $\mu_{p q}=\left\langle\phi_{p}|\vec{\mu}| \phi_{q}\right\rangle$. Then, starting from the reduction of the many particle transition moments and neglecting the conjugate Dyson, one can arrive at the following expression

$$
D_{x}^{F I}=\sum_{q}\left\langle\phi_{\varepsilon}\left|\mu_{x}\right| \phi_{q}\right\rangle \gamma_{q} \equiv\left\langle\phi_{\varepsilon}\left|\mu_{x}\right| \phi^{d}\right\rangle, \quad \phi^{d}=\sum_{q} \gamma_{q} \phi_{q}
$$

\section{Derivation of Eq. (4)}

The derivation of Eq. 4 follows Martin and Shirley ${ }^{2}$ and Arneberg et al. ${ }^{1}$ We consider an orthonormal molecular orbital (MO) basis $\left\{\phi_{p}\right\}$ (of the initial state) with associated creation $a_{p}^{\dagger}$ and annihilation $a_{p}$ operators. We can expand the photoelectron function $\phi_{\epsilon}$ on this basis

$$
\phi_{\epsilon}=\sum_{p}\left\langle\phi_{p} \mid \phi_{\epsilon}\right\rangle \phi_{p}
$$


Whereby the corresponding creation and annihilation operators become,

$$
a_{\epsilon}^{\dagger}=\sum_{p}\left\langle\phi_{p} \mid \phi_{\epsilon}\right\rangle a_{p}^{\dagger} ; \quad a_{\epsilon}=\sum_{p}\left\langle\phi_{\epsilon} \mid \phi_{p}\right\rangle a_{p}
$$

Moreover

$$
\left[a_{p}, a_{q}^{\dagger}\right]_{+}=\delta_{p q} ; \quad\left[a_{\epsilon}, a_{q}^{\dagger}\right]_{+}=\left\langle\phi_{\epsilon} \mid \phi_{q}\right\rangle
$$

From Eqs. (3) and (6), we can then write the transition dipole moment as

$$
D^{F I}=\left\langle a_{\epsilon}^{\dagger} \Psi_{F}^{N-1}|\hat{\mu}| \Psi_{I}^{N}\right\rangle=\left\langle\Psi_{F}^{N-1}\left|a_{\epsilon} \hat{\mu}\right| \Psi_{I}^{N}\right\rangle=\sum_{p q} \mu_{p q}\left\langle\Psi_{F}^{N-1}\left|a_{\epsilon} a_{p}^{\dagger} a_{q}\right| \Psi_{I}^{N}\right\rangle
$$

Using Eqs. (6) and (7) we rewrite

$$
\begin{aligned}
a_{\epsilon} a_{p}^{\dagger} a_{q} & =\left\langle\phi_{\epsilon} \mid \phi_{p}\right\rangle a_{q}-a_{p}^{\dagger} a_{\epsilon} a_{q}=\left\langle\phi_{\epsilon} \mid \phi_{p}\right\rangle a_{q}+a_{p}^{\dagger} a_{q} a_{\epsilon} ; \\
a_{\epsilon} \hat{\mu} & =\sum_{p q} \mu_{p q} a_{\epsilon} a_{p}^{\dagger} a_{q} \\
& =\sum_{p q} \mu_{p q}\left\langle\phi_{\epsilon} \mid \phi_{p}\right\rangle a_{q}-\sum_{p q} \mu_{p q} a_{p}^{\dagger} a_{\epsilon} a_{q} \\
& =\sum_{q} \sum_{p}\left\langle\phi_{\epsilon} \mid \phi_{p}\right\rangle\left\langle\phi_{p}|\vec{\mu}| \phi_{q}\right\rangle a_{q}+\left(\sum_{p q} \mu_{p q} a_{p}^{\dagger} a_{q}\right) a_{\epsilon} \\
& =\sum_{q}\left\langle\phi_{\epsilon}\left|\left(\sum_{p}\left|\phi_{p}\right\rangle\left\langle\phi_{p}\right|\right) \vec{\mu}\right| \phi_{q}\right\rangle a_{q}+\hat{\mu} a_{\epsilon} \\
& =\sum_{q}\left\langle\phi_{\epsilon}|\vec{\mu}| \phi_{q}\right\rangle a_{q}+\hat{\mu} a_{\epsilon} \\
& =\sum_{q}\left\langle\phi_{\epsilon}|\mu| \phi_{q}\right\rangle a_{q}+\hat{\mu} \sum_{q}\left\langle\phi_{\epsilon} \mid \phi_{q}\right\rangle a_{q}
\end{aligned}
$$


and finally arrive at

$$
\begin{aligned}
D^{F I} & =\sum_{q}\left\langle\phi_{\epsilon}|\mu| \phi_{q}\right\rangle\left\langle\Psi_{F}^{N-1}\left|a_{q}\right| \Psi_{I}^{N}\right\rangle+\sum_{q}\left\langle\phi_{\epsilon} \mid \phi_{q}\right\rangle\left\langle\Psi_{F}^{N-1}\left|\hat{\mu} a_{q}\right| \Psi_{I}^{N}\right\rangle \\
& =\sum_{q}\left\langle\phi_{\epsilon}|\mu| \phi_{q}\right\rangle \gamma_{q}^{F}+\sum_{q}\left\langle\phi_{\epsilon} \mid \phi_{q}\right\rangle \eta_{q}^{F}
\end{aligned}
$$

where we have introduced the definitions of the following amplitudes (expansion coefficients)

$$
\gamma_{q}^{F}=\left\langle\Psi_{F}^{N-1}\left|a_{q}\right| \Psi_{I}^{N}\right\rangle ; \quad \eta_{q}^{F}=\left\langle\Psi_{F}^{N-1}\left|\hat{\mu} a_{q}\right| \Psi_{I}^{N}\right\rangle
$$

The first term in Eq. (11) is called the direct term, and the second one the conjugate term. According to Eq. (11), the full transition moment is given by 1-particle matrix elements with the continuum, multiplied by the $\gamma_{q}^{F}$ and $\eta_{q}^{F}$ amplitudes, which are matrix elements of the initial and final bound states. This is sufficient to get the cross section for a single channel calculation, with a product structure of the final wavefunction.

Introducing a resolution of identity on a set of $(N-1)$-electron wavefunctions into the conjugate term, we obtain

$$
\begin{aligned}
\left\langle\Psi_{F}^{N-1}\left|\hat{\mu} a_{q}\right| \Psi_{I}^{N}\right\rangle & =\sum_{J}\left\langle\Psi_{F}^{N-1}|\mu| \Psi_{J}^{N-1}\right\rangle\left\langle\Psi_{J}^{N-1}\left|a_{q}\right| \Psi_{I}^{N}\right\rangle \\
\eta_{q}^{F} & =\sum_{J} D_{N-1}^{F J} \gamma_{q}^{J}
\end{aligned}
$$

where $D_{N-1}^{F J}$ indicates the transition dipole moment between two electronic states of the $(N-1)$-electron ionic (bound) system. At high energy, or simply when the photoelectron wavefunction is considered orthogonal to all bound orbitals included in the expansion of the initial state, the conjugate term is negligible, and only the direct term survives.

Finally, introducing in the direct term the definition of the Dyson orbital, $\phi^{d}$ :

$$
\phi^{d}=\sum_{q} \phi_{q} \gamma_{q}^{F}
$$


we arrive at the expression for the transition moment already given in Eq. (4)

$$
D_{x}^{F I}=\sum_{q}\left\langle\phi_{\varepsilon}\left|\mu_{x}\right| \phi_{q}\right\rangle \gamma_{q}^{F} \equiv\left\langle\phi_{\varepsilon}\left|\mu_{x}\right| \phi^{d}\right\rangle
$$

At the independent particle level (unrelaxed Hartree-Fock configurations), we have that $\gamma_{q}=1$ for the primary ionic states, and $\gamma_{q}=0$ otherwise, i.e.

$$
\left|\Psi_{F}^{N-1}\right\rangle=a_{q}|\mathrm{HF}\rangle
$$

Thus, those are the only final states that are allowed, and their corresponding Dyson orbitals are simply the canonical occupied Hartree-Fock (HF) orbitals $\phi_{p}$, with unit norm.

The square norm of the Dyson orbital

$$
R_{F} \equiv\left\|\phi^{d}\right\|^{2}=\sum_{p}\left|\gamma_{p}^{F}\right|^{2}
$$

is often called spectral strength or pole strength of the final bound state.

Correlation lowers the spectral strengths of the primary ionic states, and gives intensity to additional states (satellite or shake-up states) characterized by additional electronic excitations. The usual values for the outermost primary states (outer valence) are about 0.8-1.00. The effects become especially strong for the inner valence region (higher ionic states of the same symmetry) mainly because of the mixing with $2 \mathrm{~h}-1 \mathrm{p}$ configurations relative to outer excitations, and the spectral strength can spread over many final states of low intensity. Another important effect is the mixing of different canonical MOs in the Dyson orbital, due to rotation of the occupied orbitals upon ionization. 


\section{Photoelectron transition strength in the EOM-CC formalism}

In coupled cluster (CC) theory, a transition strength (matrix element) between two states $I$ and $F$ is written as

$$
S_{x y}^{I F}=\frac{1}{2}\left\{D_{x}^{I F} D_{y}^{F I}+\left(D_{y}^{I F} D_{x}^{F I}\right)^{*}\right\}
$$

where the left and right moments are,

$$
D_{x}^{I F}=\left\langle\Psi_{I}\left|\mu_{x}\right| \Psi_{F}\right\rangle^{(L)} ; \quad D_{x}^{F I}=\left\langle\Psi_{F}\left|\mu_{x}\right| \Psi_{I}\right\rangle^{(R)}
$$

The labels $(L)$ or $(R)$ are used to highlight that, in EOM-CC, the above are not true scalar products, since they are not Hermitian, $D_{x}^{F I} \neq\left(D_{x}^{I F}\right)^{*}$. We can then write

$$
\begin{array}{ll}
D_{x}^{I F}=\left\langle\left(\phi_{f i}^{d}\right)^{L}\left|\mu_{x}\right| \phi_{\epsilon}\right\rangle ; & \phi_{f i}^{L}=\sum_{p} \gamma_{p}^{L} \phi_{p} \\
D_{x}^{F I}=\left\langle\phi_{\epsilon}\left|\mu_{x}\right|\left(\phi_{f i}^{d}\right)^{R}\right\rangle & \phi_{f i}^{R}=\sum_{p} \gamma_{p}^{R} \phi_{p}
\end{array}
$$

From now onwards, we will use $\phi_{f i}^{R}$ instead of $\left(\phi_{f i}^{d}\right)^{R}$ and similarly for the left one. The transition moments in Eq. (20) are now true scalar products (as computed in our program) between the continuum and the left and right Dyson orbitals. Using the above expressions, we then consider the photoelectron transition strength matrix

$$
\begin{aligned}
S_{x y}^{F I} & \equiv\left\langle\Psi_{F}\left|\mu_{x}\right| \Psi_{I}\right\rangle\left\langle\Psi_{I}\left|\mu_{y}\right| \Psi_{F}\right\rangle \\
& =\frac{1}{2}\left[\left(\left\langle\phi_{\epsilon}\left|\mu_{x}\right| \phi_{f i}^{R}\right\rangle\left\langle\phi_{f i}^{L}\left|\mu_{y}\right| \phi_{\epsilon}\right\rangle\right)+\left(\left\langle\phi_{\epsilon}\left|\mu_{y}\right| \phi_{f i}^{R}\right\rangle\left\langle\phi_{f i}^{L}\left|\mu_{x}\right| \phi_{\epsilon}\right\rangle\right)^{*}\right] \\
& =\frac{1}{2}\left[\left\langle\phi_{\epsilon}\left|\mu_{x}\right| \phi_{f i}^{R}\right\rangle\left\langle\phi_{\epsilon}\left|\mu_{y}\right| \phi_{f i}^{L}\right\rangle^{*}+\left\langle\phi_{\epsilon}\left|\mu_{x}\right| \phi_{f i}^{L}\right\rangle\left\langle\phi_{\epsilon}\left|\mu_{y}\right| \phi_{f i}^{R}\right\rangle^{*}\right]
\end{aligned}
$$

Thus, in practice, any expression (in the original code for variational wavefunction) that contains Hermitian products of two dipole matrix elements is transformed (in the non-variational 
CC case) as

$$
D_{\alpha}^{F I}\left(D_{\beta}^{F I}\right)^{*} \rightarrow \frac{1}{2}\left\{D_{R, \alpha}^{F I} D_{L, \beta}^{I F *}+D_{L, \alpha}^{I F} D_{R, \beta}^{F I *}\right\}
$$

where $\alpha$ and $\beta$ refer to different cartesian components $x, y$ or $z$, which is the form employed in $\mathrm{Eq}(30)$.

\section{Calculation of the photoionization observables using the LCAO B- spline approach}

The differential cross section is given by,

$$
\frac{d \sigma}{d \vec{k}}=4 \pi^{2} \alpha \omega\left|\left\langle\Psi_{F}|\vec{\varepsilon} \cdot \vec{\mu}| \Psi_{i}\right\rangle\right|^{2}
$$

The terms in Eq. (25) have usual meaning as defined in the main article.

To obtain the differential cross section, we first compute the continuum orbitals in the angular momentum representation

$$
H \phi_{\epsilon, l m}=E \phi_{\epsilon, l m}
$$

with real (K-matrix) boundary conditions, as well as the transition dipole moments with left and right Dyson orbitals $\phi^{L}$ and $\phi^{R}$.

Then, $\phi_{\epsilon, l m}$ and the corresponding dipole moments are transformed to incoming-wave boundary conditions, $\phi_{\epsilon l m}^{(-)}$, and finally to $\phi_{\bar{k}}^{(-)}$, where

$$
\phi_{\bar{k}}^{(-)}=\frac{1}{\sqrt{m}} \sum_{l m} i^{l} e^{-i \sigma_{l}} Y_{l m}^{*}(\hat{k}) \phi_{\epsilon l m}^{(-)}
$$

Omitting, for ease of notation, the superscript FI, the left and right photoelectron dipole matrix elements ( in Eq. (20)) are obtained as 


$$
D_{\epsilon l m, \gamma}^{R}=\left\langle\phi_{\epsilon l m}^{(-)}\left|\mu_{\gamma}\right| \phi_{f i}^{R}\right\rangle
$$

and similarly for $D_{\epsilon l m, \gamma}^{L}$. Following standard manipulations as adopted by Chandra, ${ }^{3}$ one obtains, for randomly oriented molecules,

$$
\frac{d \sigma}{d \vec{k}}=\pi \alpha \omega(-1)^{m_{r}} \sum_{L} A_{L}(k) P_{L}(\cos \theta)
$$

where $m_{r}$ specifies the polarization of light $\left[m_{r}=0,+1,-1\right.$ for linearly polarized (LP), left circularly polarized (LCP) and right circularly polarized (RCP) light, respectively]; $\theta$ is the angle between the momentum of the electron and either the polarization vector (for LP) or the direction of propagation (for CP) of the light beam . The coefficients $A_{L}(k)$ are given by

$$
\begin{aligned}
A_{L}(k)=(2 L+1) & \left(\begin{array}{ccc}
1 & 1 & L \\
m_{r} & -m_{r} & 0
\end{array}\right) \sum_{\substack{l m \gamma \\
l^{\prime} m^{\prime} \gamma^{\prime}}}(-1)^{m+\gamma} \sqrt{(2 l+1)\left(2 l^{\prime}+1\right)} \\
& \times\left(\begin{array}{ccc}
l & l^{\prime} & L \\
0 & 0 & 0
\end{array}\right)\left(\begin{array}{ccc}
l & l^{\prime} & L \\
-m & m^{\prime} & \left(m-m^{\prime}\right)
\end{array}\right)\left(\begin{array}{ccc}
1 & 1 & L \\
\gamma^{\prime} & -\gamma & \left(\gamma-\gamma^{\prime}\right)
\end{array}\right) \\
& \times \frac{1}{2}\left(D_{\epsilon l m, \gamma}^{R} D_{\epsilon l^{\prime} m^{\prime}, \gamma^{\prime}}^{L *}+D_{\epsilon l m, \gamma}^{L} D_{\epsilon l^{\prime} m^{\prime}, \gamma^{\prime}}^{R *}\right) .
\end{aligned}
$$

Establishing the correspondences

$$
\begin{aligned}
\frac{\sigma}{4 \pi} & =\pi \alpha \omega(-1)^{m_{r}} A_{0} \\
D & =\frac{A_{1}\left(m_{r}=1\right)}{A_{0}} ; \quad \beta=\frac{A_{2}\left(m_{r}=0\right)}{A_{0}}
\end{aligned}
$$


we finally obtain the usual expression for the differential cross section,

$$
\frac{d \sigma}{d k}=\frac{\sigma}{4 \pi}\left[1+m_{r} D \cos \theta+\left(-\frac{1}{2}\right)^{m_{r}} \beta P_{2}(\cos \theta)\right]
$$

or simply, for linear polarization $\left(m_{r}=0\right)$,

$$
\frac{d \sigma}{d k}=\frac{\sigma}{4 \pi}\left[1+\beta P_{2}(\cos \theta)\right]
$$

\section{Parameters used in the LCAO B-spline code and in the ezDyson code}

Table S1: Parameters used in the LCAO B-spline code. $L_{\max }$ and $l_{\max }$ are the maximum angular momentum employed in the one center expansion at origin and on each atom (offcenter), respectively. $R_{\max }$ and $r_{\max }$ are the maximum radial grid length from origin and off-center atoms, respectively.

\begin{tabular}{l|r|r|r|c|c}
\hline Molecule & $\begin{array}{c}\# \text { grid } \\
\text { points }\end{array}$ & \multicolumn{2}{|c|}{$L_{\max }$} & $\begin{array}{c}R_{\max } \\
\text { (a.u. })\end{array}$ & \multicolumn{2}{|c}{$\begin{array}{c}\text { LCAO descriptor } \\
r_{\max }(\text { a.u. })\end{array}$} & $l_{\max }$ \\
\hline $\mathrm{Ar}$ & 125 & 2 & 25 & - & - \\
$\mathrm{CO}$ & 125 & 20 & 25 & $0.4(\mathrm{C}, \mathrm{O})$ & 2 \\
$\mathrm{CS}$ & 125 & 20 & 25 & $0.4(\mathrm{C}, \mathrm{S})$ & 2 \\
$\mathrm{H}_{2} \mathrm{O}$ & 125 & 10 & 25 & $1.0(\mathrm{H})$ & 2 \\
$\mathrm{CH}_{2} \mathrm{O}$ & 100 & 12 & 25 & $0.2(\mathrm{C}, \mathrm{O}, \mathrm{H})$ & 2 \\
$\mathrm{C}_{4} \mathrm{H}_{4} \mathrm{O}$ (planar) & 100 & 15 & 25 & $1.0(\mathrm{C}, \mathrm{O}, \mathrm{H})$ & 2 \\
$\mathrm{C}_{4} \mathrm{H}_{4} \mathrm{O}$ (bent) & 100 & 15 & 25 & $1.0(\mathrm{C}, \mathrm{O}, \mathrm{H})$ & 2 \\
$(S)-\mathrm{C}_{3} \mathrm{H}_{6} \mathrm{O}$ & 50 & 12 & 25 & $0.6(\mathrm{O}), 1.0(\mathrm{C}, \mathrm{H})$ & 2 \\
\hline
\end{tabular}


Table S2: Parameters for calculations of cross-sections and asymmetry parameter using ezDyson code. $L_{\max }$ is the maximum angular momentum number for which spherical waves are generated and the dipole matrix elements are calculated. $E_{k}^{\min }$ and $E_{k}^{\max }$ are the minimum and maximum value, respectively, of the kinetic energy for which the cross-section and asymmetry parameter are computed. $\left\langle R^{2}\right\rangle$ is the average square radial distance, used for analyzing the size and shape of the left Dyson orbital.

\begin{tabular}{l|c|c|c|c|c}
\hline Molecule & $L_{\max }$ & \# grid points & $E_{k}^{\min }(\mathrm{eV})$ & $E_{k}^{\max }(\mathrm{eV})$ & $\left\langle R^{2}\right\rangle(\AA)$ \\
\hline $\mathrm{Ar}$ & 2 & 101 & 0.1 & 100.1 & 0.608698 \\
$\mathrm{CO}$ & 5 & 101 & 0.1 & 100.1 & 1.052489 \\
$\mathrm{CS}$ & 5 & 101 & 0.1 & 100.1 & 1.697160 \\
$\mathrm{H}_{2} \mathrm{O}$ & 5 & 50 & 0.5 & 50.5 & 0.763224 \\
$\mathrm{CH}_{2} \mathrm{O}$ & 5 & 101 & 0.1 & 100.1 & 1.121095 \\
\hline
\end{tabular}

\section{Ionization energies (IE) and Dyson norms}

Table S3: IE (in eV) and EOM-CCSD Dyson square norms of the ground state using augcc-pVTZ basis set. Experimental results are taken from Ref. 4 and 5. Mulliken's symmetry convention is assumed.

\begin{tabular}{l|c|c|c|c|c}
\hline System & Irrep & $\begin{array}{c}\text { KT } \\
\text { IE }(\mathrm{eV})\end{array}$ & $\begin{array}{c}\text { EOM-CCSD } \\
\text { IE }(\mathrm{eV})\end{array}$ & $\begin{array}{c}\text { Dyson sq. norm } \\
\left(\left|\phi^{L} \cdot \phi^{R}\right|^{2}\right)\end{array}$ & $\begin{array}{c}\text { Exp. } \\
\mathrm{IE}(\mathrm{eV})\end{array}$ \\
\hline \multirow{2}{*}{$\mathrm{Ar}$} & $3 \mathrm{~s}$ & 34.83 & 29.91 & 0.859 & 29.2 \\
& $3 \mathrm{p}$ & 16.05 & 15.66 & 0.965 & 15.8 \\
\hline \multirow{2}{*}{$\mathrm{CO}$} & $5 \sigma$ & 15.10 & 14.20 & 0.952 & 14.0 \\
& $4 \sigma$ & 21.91 & 19.82 & 0.934 & 19.7 \\
& $1 \pi$ & 17.41 & 17.10 & 0.949 & 16.9 \\
\hline $\mathrm{CS}$ & $7 \sigma$ & 12.82 & 11.52 & 0.931 & 11.3 \\
& $2 \pi$ & 12.60 & 13.06 & 0.953 & 12.8 \\
\hline \multirow{2}{*}{$\mathrm{H}_{2} \mathrm{O}$} & $3 \mathrm{a}_{1}$ & 15.92 & 14.83 & 0.957 & 14.7 \\
& $1 \mathrm{~b}_{2}$ & 19.51 & 19.00 & 0.962 & 18.5 \\
& $1 \mathrm{~b}_{1}$ & 13.88 & 12.62 & 0.956 & 12.6 \\
\hline \multirow{2}{*}{$\mathrm{CH}_{2} \mathrm{O}$} & $5 \mathrm{a}_{1}$ & 17.77 & 16.15 & 0.944 & 16.0 \\
& $2 \mathrm{~b}_{2}$ & 12.05 & 10.86 & 0.951 & 10.8 \\
& $1 \mathrm{~b}_{1}$ & 14.64 & 14.66 & 0.944 & 14.5 \\
\hline $\mathrm{C}_{4} \mathrm{H}_{4} \mathrm{O}$ & $1 \mathrm{a}_{2}$ & 8.68 & 9.04 & 0.950 & 8.8 \\
$($ planar $)$ & $2 \mathrm{~b}_{1}$ & 10.80 & 10.40 & 0.946 & 10.4 \\
\hline \multirow{3}{*}{$(S)-$} & $16 \mathrm{a}$ & 11.78 & 10.37 & 0.944 & 10.4 \\
methyl- & $15 \mathrm{a}$ & 12.17 & 11.21 & 0.951 & 11.3 \\
oxirane & $14 \mathrm{a}$ & 14.01 & 13.23 & 0.952 & 13.0 \\
& $12 \mathrm{a}$ & 14.37 & 13.44 & 0.952 & 13.6 \\
& $11 \mathrm{a}$ & 15.97 & 14.40 & 0.953 & 14.5 \\
\hline
\end{tabular}




\section{Partial cross-sections and asymmetry parameters}

Argon
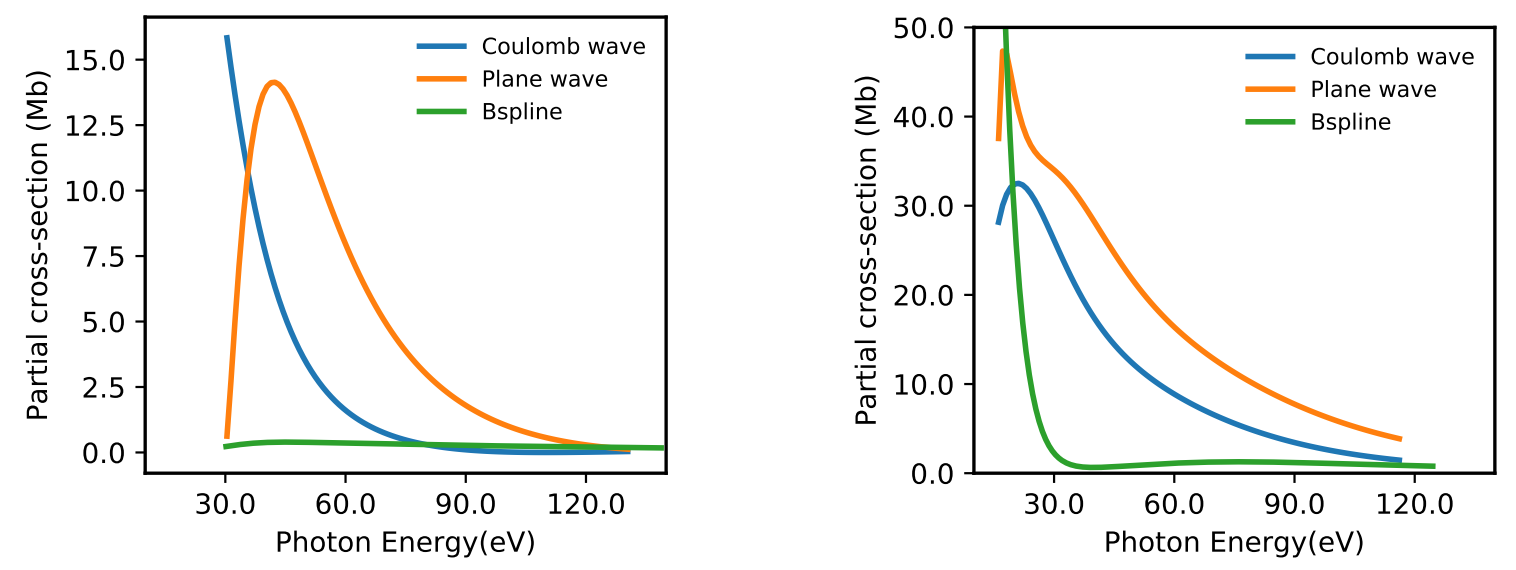

Figure S1: Partial cross-section of Ar relative to 3s (left) and 3p (right) states calculated with plane wave, coulomb wave and DFT-B-spline functions to represent the outgoing electron coupled with EOM-CCSD/aug-cc-pVTZ Dyson orbitals.

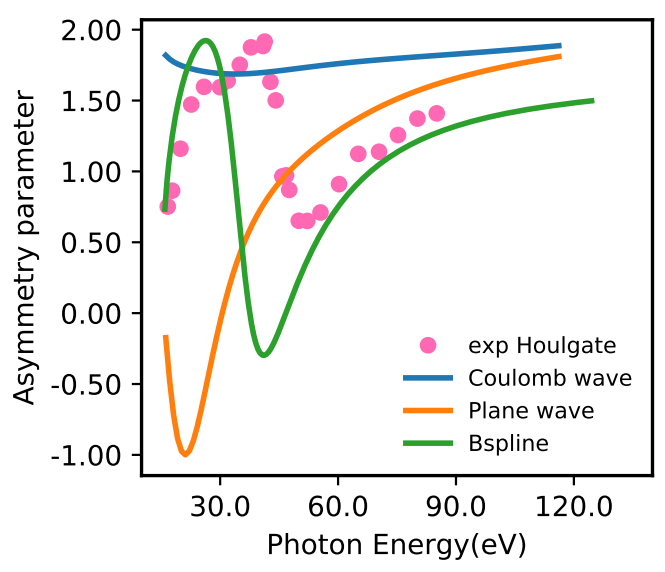

Figure S2: Comparative study of asymmetry parameter of Ar relative to ionization from 3p orbital. EOM-CCSD Dyson orbitals are used throughout.Experimental result taken from Ref. 6 . 


\section{Carbon sulphide, CS}
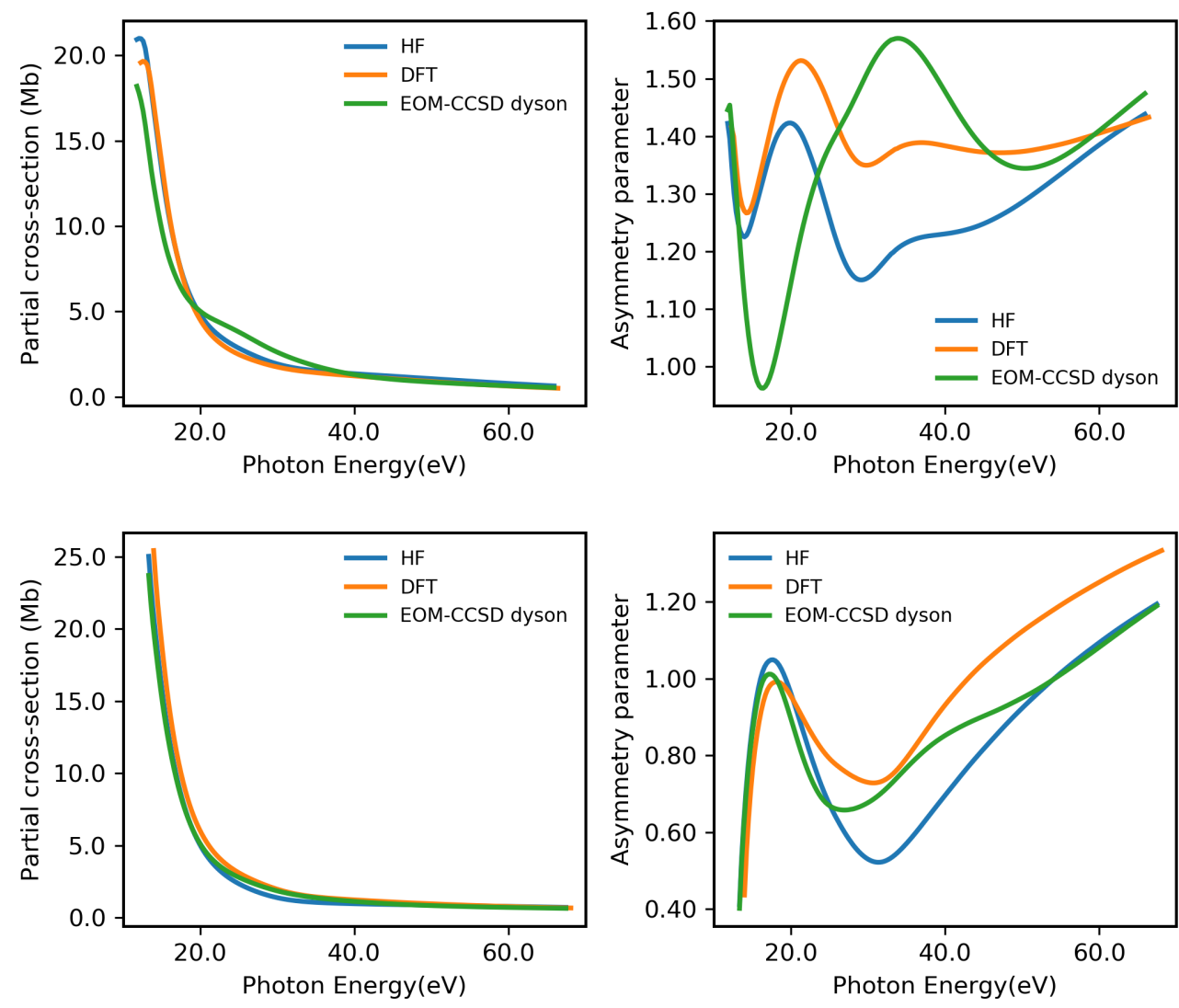

Figure S3: Partial cross-section and asymmetry parameters of CS relative to $7 \sigma$ (top) and $2 \pi$ (bottom) states calculated with HF, DFT and EOM-CCSD methods. Multicenter B-spline functions are used to describe the outgoing electron. 

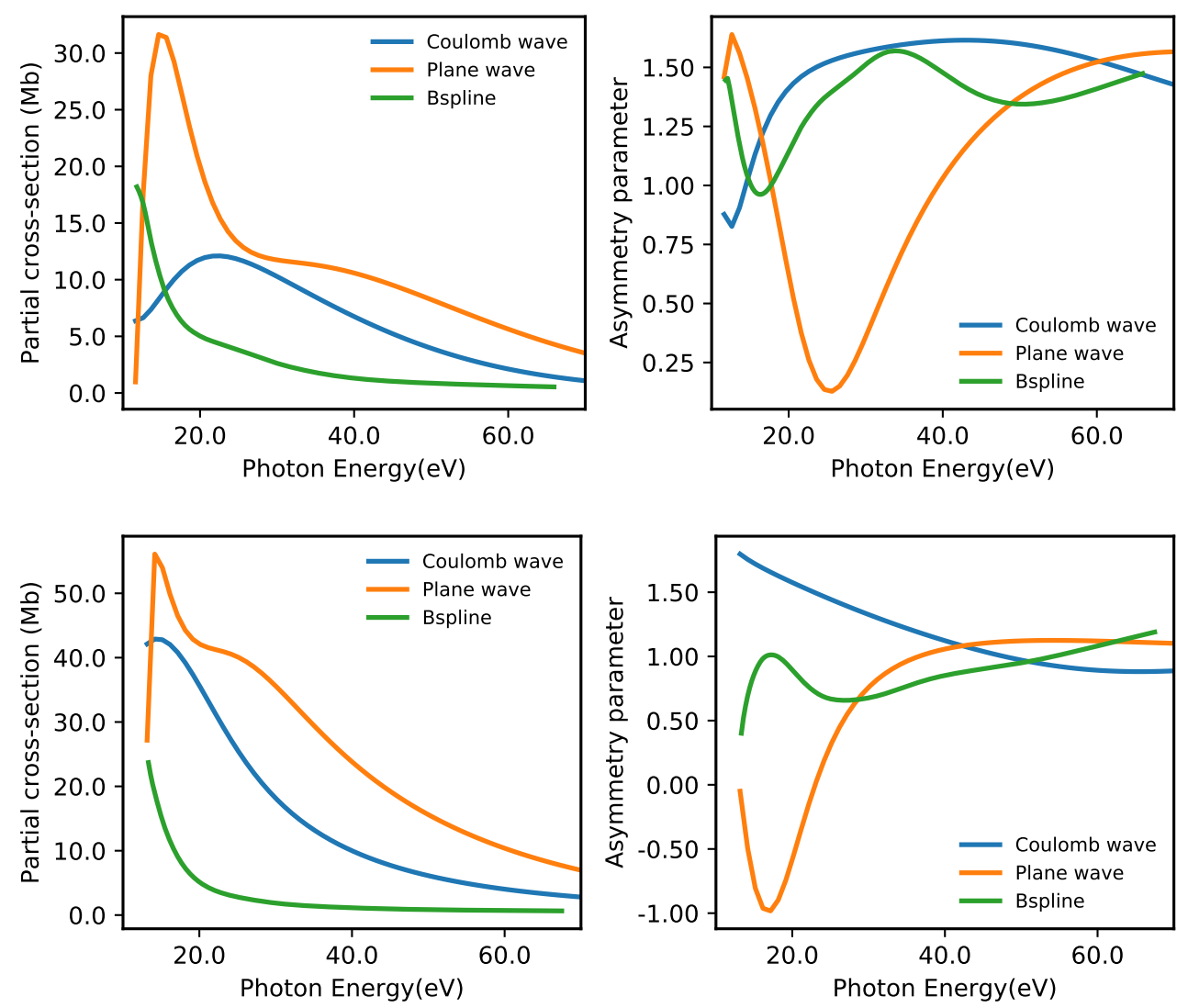

Figure S4: Partial cross-section and asymmetry parameters of CS relative to $7 \sigma$ (top) and $2 \pi$ (bottom) states calculated with plane wave, coulomb wave and B-spline functions to represent the outgoing electron coupled with EOM-CCSD/aug-cc-pVTZ Dyson orbitals. 


\section{Formaldehyde}
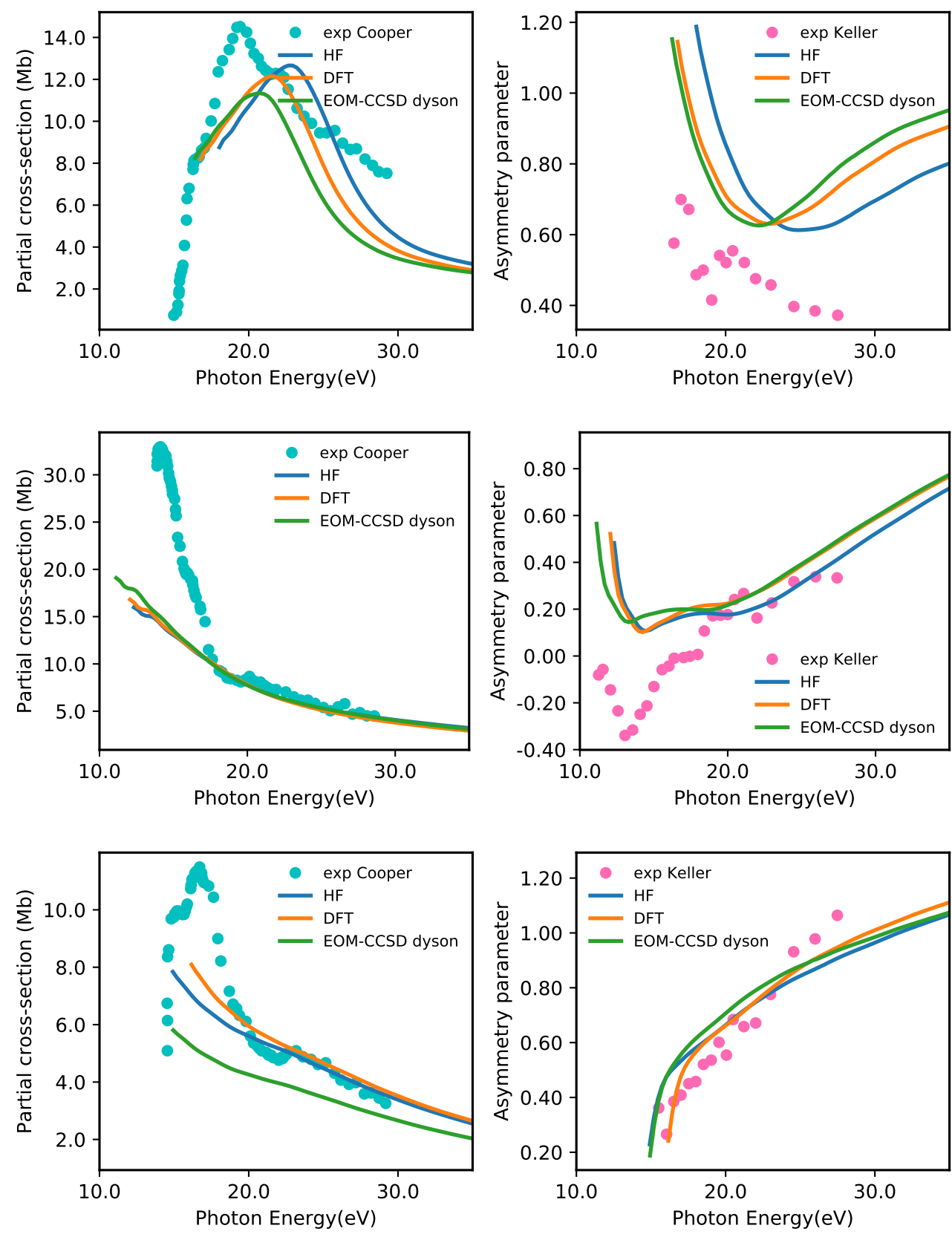

Figure S5: Partial cross-section and asymmetry parameters of $\mathrm{CH}_{2} \mathrm{O}$ relative to $5 \mathrm{a}_{1}$ (top) and $2 \mathrm{~b}_{2}$ (middle) and $1 \mathrm{~b}_{1}$ (bottom) states calculated with HF, DFT and EOM-CCSD methods. Multicenter B-spline functions are used to describe the outgoing electron. Experimental results are taken from Ref. 7 and 8. 

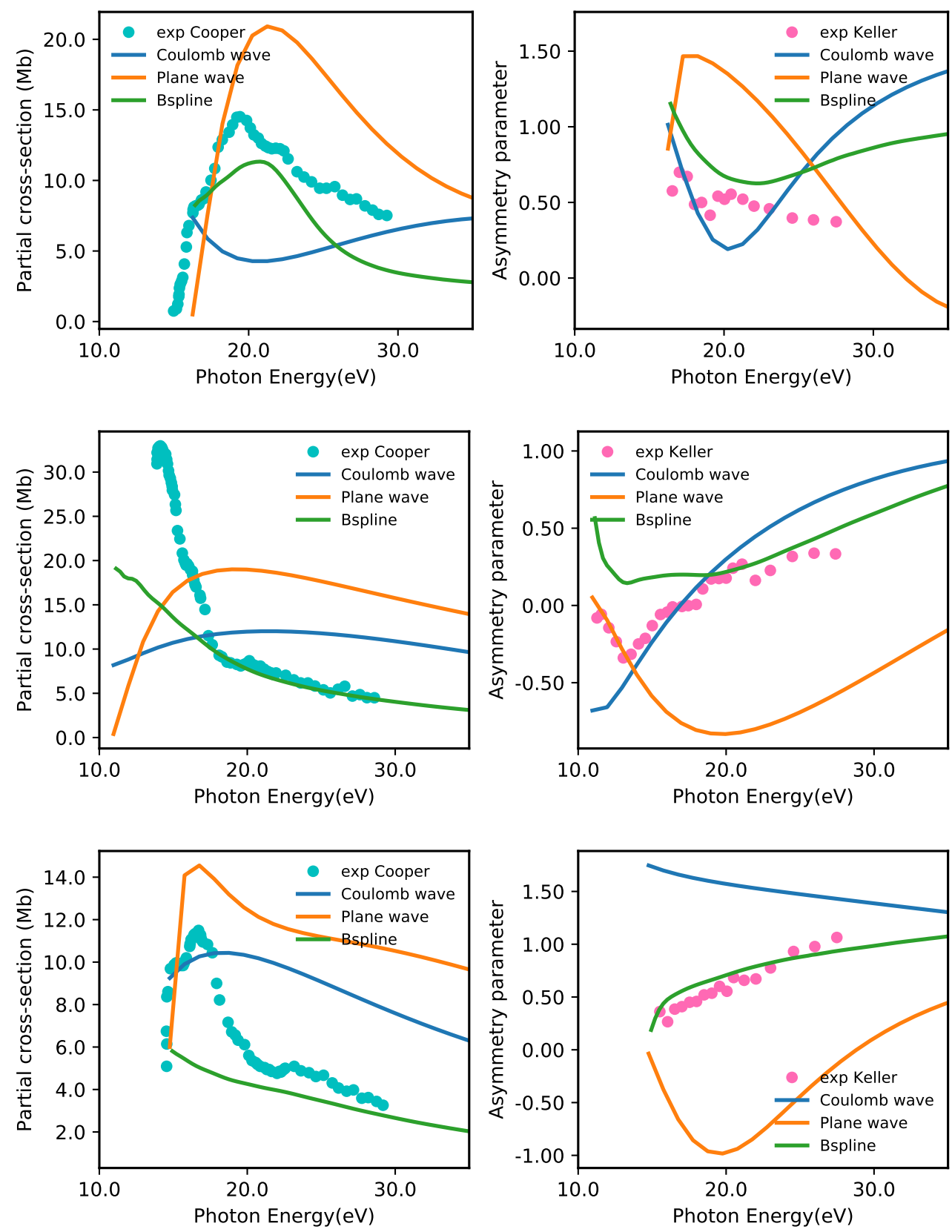

Figure S6: Partial cross-section and asymmetry parameters of $\mathrm{CH}_{2} \mathrm{O}$ relative to $5 \mathrm{a}_{1}$ (top) and $2 \mathrm{~b}_{2}$ (middle) and $1 \mathrm{~b}_{1}$ (bottom) states calculated with plane wave, coulomb wave and Bspline functions to represent the outgoing electron coupled with EOM-CCSD/aug-cc-pVTZ Dyson orbitals. Experimental results are taken from Ref. 7 and 8. 


\section{Excitation and ionization energies of furan}

Table S4: Excitation energies of furan in eV. Experimental result are from Ref. 5. CASSCF, NEVPT2, TDDFT and ADC(2) results are from Ref. 9.

\begin{tabular}{c|c|c|c|c|c|c|c}
\hline Geometry & State & EOM-CCSD & CAS-[6,9] & NEVPT2 & TDDFT & ADC(2) & Exp. \\
\hline \multirow{2}{*}{ Planar } & ${ }^{1} \mathrm{~A}_{2}\left(\mathrm{~S}_{1}\right)$ & 6.15 & 5.71 & 5.98 & 5.51 & 5.91 & 6.04 \\
& ${ }^{1} \mathrm{~B}_{2}\left(\mathrm{~S}_{2}\right)$ & 6.47 & 6.91 & 6.63 & 5.98 & 6.44 & 6.49 \\
\hline \multirow{2}{*}{ Bent } & ${ }^{1} \mathrm{~A}$ & 2.11 & - & - & - & - & - \\
& ${ }^{1} \mathrm{~A}$ & 3.85 & - & - & - & - & - \\
\hline
\end{tabular}

Table S5: Ionization energy from the excited state of furan in eV. CASSCF results are from Ref. 9

\begin{tabular}{c|c|c|c|c} 
Geometry & Excited state & Ionized state of cation & EOM-IP-CCSD & CAS-[6,9] \\
\hline \multirow{2}{*}{ Planar } & ${ }^{1} \mathrm{~A}_{2}\left(\mathrm{~S}_{1}\right)$ & ${ }^{2} \mathrm{~A}_{2}$ & 2.89 & 2.43 \\
& ${ }^{1} \mathrm{~B}_{2}\left(\mathrm{~S}_{2}\right)$ & ${ }^{2} \mathrm{~A}_{2}$ & 2.57 & 1.23 \\
\hline \multirow{2}{*}{ Bent } & ${ }^{1} \mathrm{~A}\left(\mathrm{~S}_{1}\right)$ & ${ }^{2} \mathrm{~A}$ & 5.23 & 4.22 \\
& ${ }^{1} \mathrm{~A}\left(\mathrm{~S}_{2}\right)$ & ${ }^{2} \mathrm{~A}$ & 3.48 & 2.96 \\
\hline
\end{tabular}




\section{Excited state cross-section and asymmetry parameter of furan at bent geometry}
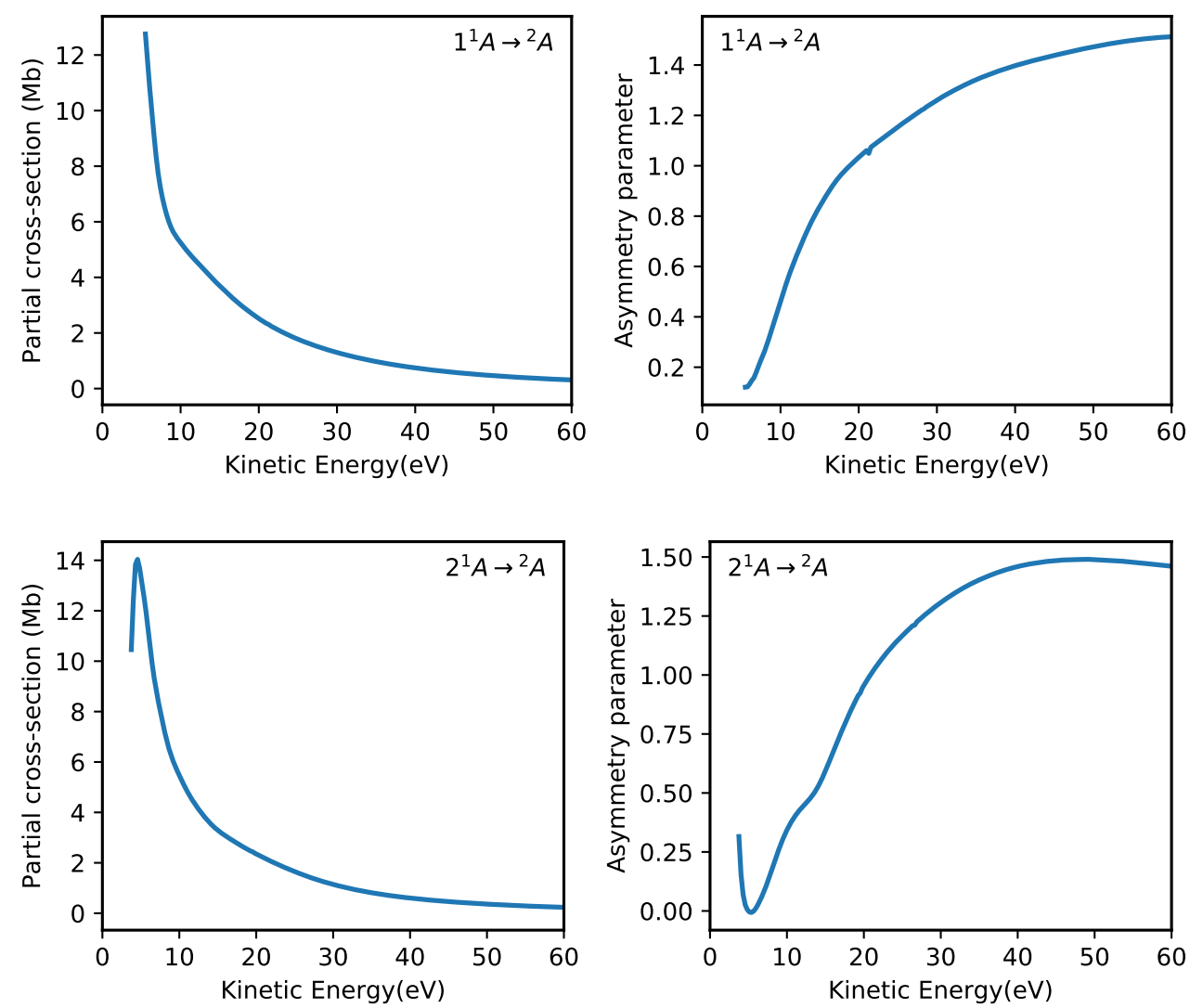

Figure S7: Computed photoionization partial cross section (left) and photoelectron asymmetry parameter (right) for transition from $S_{1}$ (top) and $S_{2}$ (bottom) states of furan at a puckered geometry. 


\section{Cartesian coordinates of all molecules}
CO (Angs)
$0 \quad 0.00000000$
0.00000000
0.48356580
C $\quad 0.00000000$
0.00000000
$-0.64475440$
$\mathbf{H}_{2} \mathbf{O}$ (Angs)
$0 \quad-0.00000000$
$-0.00000000$
0.11730000
H $\quad-0.75720000 \quad-0.00000000$
$-0.46920000$
H $\quad 0.75720000$
0.00000000
$-0.46920000$
CS (Angs)
S $\quad 0.00000000$
0.00000000
0.41860909
C $\quad 0.00000000$
0.00000000
$-1.11629091$

$\mathrm{CH}_{2} \mathrm{O}$ (Angs)

$\begin{array}{lrrr}\text { O } & 0.00000000 & 0.00000000 & 0.67595000 \\ \text { C } & 0.00000000 & 0.00000000 & -0.52905000 \\ \text { H } & 0.94290000 & 0.000000000 & -1.11665000 \\ \text { H } & -0.94290000 & -0.000000000 & -1.11665000\end{array}$

$\mathrm{C}_{4} \mathrm{H}_{4} \mathrm{O}$ (Angs)-Planar

$\begin{array}{llll}0 & 0.00000000 & 0.00000000 & 1.15570089\end{array}$

$\begin{array}{llll}\text { C } & -0.71808000 & 0.00000000 & -0.96094011\end{array}$

$\begin{array}{llll}\text { C } & 0.71808000 & 0.00000000 & -0.96094011\end{array}$

$\begin{array}{llll}\text { C } & -1.09300200 & 0.00000000 & 0.35220389\end{array}$

$\begin{array}{llll}\text { C } & 1.09300200 & 0.00000000 & 0.35220389\end{array}$ 


$$
\begin{array}{lrrr}
\text { H } & 2.05718500 & 0.00000000 & 0.85420589 \\
\text { H } & -2.05718500 & 0.00000000 & 0.85420589 \\
\text { H } & 1.37995400 & 0.00000000 & -1.82459211 \\
\text { H } & -1.37995400 & 0.00000000 & -1.82459211
\end{array}
$$

$\mathrm{C}_{4} \mathrm{H}_{4} \mathrm{O}$ (Angs)- Bent

$\begin{array}{lrrr}\text { O } & 1.09645392 & -0.24983976 & -0.34616477 \\ \text { C } & 0.64775401 & 0.90476604 & 0.14769665 \\ \text { C } & -0.87836149 & 0.84558708 & -0.05929252 \\ \text { C } & -1.07423176 & -0.47773249 & -0.13303448 \\ \text { C } & 0.19506068 & -1.05871585 & 0.15814657 \\ \text { H } & 1.19377197 & 1.61166861 & 0.79100107 \\ \text { H } & -1.51941099 & 1.72028223 & 0.01907603 \\ \text { H } & -1.97177413 & -1.14269353 & 0.02025521 \\ \text { H } & 0.18445315 & -1.47396792 & 1.25788853\end{array}$

$\begin{array}{lrrr}\mathrm{C}_{3} \mathrm{H}_{6} \mathrm{O}(\mathrm{au}) & & \\ \mathrm{C} & -0.28956361 & 0.08176958 & 0.92701577 \\ \mathrm{C} & 1.93885200 & -1.15600766 & -0.10800484 \\ \mathrm{C} & -2.79852375 & -0.19094696 & -0.27656907 \\ \mathrm{O} & 1.53613696 & 1.47730615 & -0.46998636 \\ \mathrm{H} & 1.71860882 & -2.32670888 & -1.80795335 \\ \mathrm{H} & 3.52273536 & -1.64526642 & 1.13875511 \\ \mathrm{H} & -0.27744501 & 0.50560530 & 2.96417187 \\ \mathrm{H} & -2.56905279 & -0.59002862 & -2.30639911 \\ \mathrm{H} & -3.89960425 & 1.56511541 & -0.07933409 \\ \mathrm{H} & -3.88892566 & -1.73605572 & 0.59599929\end{array}$




\section{References}

(1) Arneberg, R.; Müller, J.; Manne, R. Configuration interaction calculations of satellite structure in photoelectron spectra of $\mathrm{H}_{2} \mathrm{O}$. Chem. Phys. 1982, 64, $249-258$.

(2) Martin, R. L.; Shirley, D. A. Theory of core-level photoemission correlation state spectra. J. Chem. Phys. 1976, 64, 3685-3689.

(3) Chandra, N. Photoelectron spectroscopic studies of polyatomic molecules. I. Theory. J. Phys. B: At. Mol. Phys. 1987, 20, 3405-3415.

(4) Jonathan, N.; Morris, A.; Okuda, M.; Ross, K. J.; Smith, D. J. Photoelectron spectroscopy of transient species. The CS molecule. Faraday Discuss. Chem. Soc. 1972, 54, $48-55$.

(5) Kimura, K.; Kimura, ., Katsumi Handbook of HeI photoelectron spectra of fundamental organic molecules : ionization energies, ab initio assignments, and valence electronic structure for 200 molecules; Tokyo : Japan Scientific Societies Press ; New York : Halsted Press, 1981; Includes indexes.

(6) Houlgate, R.; West, J.; Codling, K.; Marr, G. The angular distribution of the 3p electrons and the partial cross section of the 3 s electrons of argon from threshold to $70 \mathrm{eV} . J$ Electron Spectros Relat Phenomena 1976, 9, 205 - 209.

(7) Cooper, G.; Anderson, J. E.; Brion, C. Absolute photoabsorption and photoionization of formaldehyde in the VUV and soft X-ray regions (3-200 eV). Chem. Phys. 1996, 209, $61-77$.

(8) Keller, P.; Taylor, J.; Grimm, F.; Carlson, T. A. Angle-resolved photoelectron spectroscopy of formaldehyde and methanol. Chem. Phys. 1984, 90, $147-153$.

(9) Ponzi, A.; Sapunar, M.; Angeli, C.; Cimiraglia, R.; Došlić, N.; Decleva, P. Photoioniza- 
tion of furan from the ground and excited electronic states. J. Chem. Phys. 2016, 144, 084307. 
